\title{
Efficient calculation of crossing symmetric BCJ tree numerators
}

\author{
Alex Edison and Fei Teng \\ Department of Physics and Astronomy, Uppsala University, \\ Uppsala SE-75108, Sweden \\ E-mail: alexander.edison@physics.uu.se, fei.teng@physics.uu.se
}

ABSTRACT: In this paper, we develop an improved method for directly calculating doublecopy-compatible tree numerators in (super-)Yang-Mills and Yang-Mills-scalar theories. Our new scheme gets rid of any explicit dependence on reference orderings, restoring a form of crossing symmetry to the numerators. This in turn improves the computational efficiency of the algorithm, allowing us to go well beyond the number of external particles accessible with the reference order based methods. Motivated by a parallel study of one-loop BCJ numerators from forward limits, we explore the generalization to include a pair of fermions. To improve the accessibility of the new algorithm, we provide a MATHEMATICA package that implements the numerator construction. The structure of the computation also provides for a straightforward introduction of minimally-coupled massive particles potentially useful for future computations in both classical and quantum gravity.

KEYwords: Scattering Amplitudes, Gauge Symmetry, Superstrings and Heterotic Strings

ArXiv EPrint: 2005.03638 


\section{Contents}

1 Introduction 1

2 CHY integrands and baseline expansion 5

2.1 Super-Yang-Mills (sYM) theories 5

2.2 Yang-Mills-scalar $\quad 7$

3 DDM basis numerators from CHY integrands 11

3.1 Spanning trees and reference orderings 11

3.2 Recursive constructions without reference orderings for pure gluon and two$\begin{array}{ll}\text { fermion numerators } & 13\end{array}$

$\begin{array}{lll}3.3 & \text { Four-point YM example } & 18\end{array}$

$\begin{array}{lll}3.4 & \text { Recursive constructions for multi-trace } & 19\end{array}$

3.5 Multi-trace example 21

$\begin{array}{lll}4 & \text { Introducing masses } & 23\end{array}$

5 Conclusion and outlook $\quad 24$

$\begin{array}{lll}\text { A CHY integrands and scattering amplitudes } & 25\end{array}$

B Mathematica package $\quad 28$

B.1 Constituent objects 28

$\begin{array}{lll}\text { B.2 Construction functions } & 29\end{array}$

\section{Introduction}

The efficient calculation of scattering amplitudes at high precision relies on the discovery of novel methods and structures, many of which are shared between disparate theories. While these structures are often highly obscured by traditional Lagrangian/Feynman diagram approaches, they tend to lead to new formulations of amplitudes in quantum field theories.

One powerful structure in tree amplitudes is the Bern-Carrasco-Johansson (BCJ) colorkinematics duality [1-3], which allows a large class of theories to be constructed as the double copy of simpler theories. This duality has been used to push the envelop on accessible calculations in various theories of quantum gravity [4-7] by "squaring" (super-)Yang-Mills (sYM) theory. Additionally, BCJ-compatible trees have been used in the blossoming application of scattering amplitudes techniques to black hole physics in classical gravity [8-23]. Unfortunately, most textbook methods for calculating tree amplitudes do not directly generate color-kinematics dual representations. 
The Cachazo-He-Yuan (CHY) formalism [24-26] is a powerful method for studying the properties of tree-level scattering amplitudes in many theories, in part because it manifests double-copy properties. The formalism explicitly constructs tree amplitudes from the product of two half-integrands. There is now a well-developed program for exploring the types of objects needed in each half-integrand to generate theories of interest [27-34], which can be derived from world-sheet models based on ambitwistor strings [35-39] and their deformation [40,41]. The half-integrands are known to be expressible completely in Del Duca-Dixon-Maltoni half-ladder basis [42] on the support of scattering equations [26], ${ }^{1}$

$$
\mathcal{I}_{n} \stackrel{\mathrm{SE}}{=} \sum_{\beta \in S_{n-2}} \operatorname{PT}(1, \beta, n) N(1, \beta, n), \text { where } \operatorname{PT}(\rho) \equiv \frac{1}{\sigma_{\rho_{1} \rho_{2}} \sigma_{\rho_{2} \rho_{3}} \cdots \sigma_{\rho_{n-1} \rho_{n}} \sigma_{\rho_{n} \rho_{1}}} .
$$

In this basis, the kinematic coefficients $N(1, \beta, n)$ of the worldsheet Parke-Taylor factors $\mathrm{PT}(1, \beta, n)$ can be seen as master numerators, since all non-ladder kinematic numerators can be built using BCJ numerator relations on the half-ladders [49]. Due to this fact, the half-ladder numerators fully specify tree-level amplitudes. This can be explicitly seen when considering sYM through the use of the doubly-color-ordered biadjoint scalar amplitudes $m(\alpha \mid \beta)[26,50-52]$, which combine the half-ladder numerators $N$ and the DDM color structures $c[42]$ with the appropriate signs and propagators to generate amplitudes via

$$
\mathcal{A}_{n}^{\text {tree }}=\mathscr{N} \sum_{\alpha, \beta \in S_{n-2}} c(1, \alpha, n) m(1, \alpha, n \mid 1, \beta, n) N(1, \beta, n) .
$$

These numerators can be directly double-copied with appropriate partners $\tilde{N}$ to yield various gravitational theories,

$$
\mathcal{M}_{n}^{\text {tree }}=\mathscr{N}_{\mathrm{GR}} \sum_{\alpha, \beta \in S_{n-2}} \tilde{N}(1, \alpha, n) m(1, \alpha, n \mid 1, \beta, n) N(1, \beta, n) .
$$

As amplitudes for bosonic theories, both $\mathcal{A}$ and $\mathcal{M}$ are invariant under particle exchange. The permutation invariance of $2, \ldots, n-1$ is manifest if

$$
\left.N(1, \beta, n) \equiv N(1,2, \ldots, n)\right|_{2 \rightarrow \beta_{2}, 3 \rightarrow \beta_{3}, \ldots, n-1 \rightarrow \beta_{n-1}},
$$

which we will take as our definition of crossing symmetry acting on numerators. ${ }^{2}$ The resemblance of eq. (1.3) to the Kawai-Lewellen-Tye (KLT) double-copy relation [53] provides a direct construction of $N(1, \beta, n)$ using color-ordered amplitudes and KLT momentum kernel $[54,55]$. However, the result is non-local as the numerator is a rational function of Mandelstam variables, rather than polynomial.

\footnotetext{
${ }^{1}$ This expansion also holds for worldsheet correlators in generic string theories [43-48].

${ }^{2}$ The crossing symmetry in 1 and $n$ are due in part to the BCJ relations. Since the $N$ are for halfladders, one might expect that exchanging either $1 \leftrightarrow \beta_{2}$ or $n \leftrightarrow \beta_{n-1}$ in $N(1, \beta, n)$ would yield a minus sign. However, the interplay between color and kinematic jacobis allows this property of the half-ladders to be broken or restored as needed. Of course, imposing these additional crossing symmetry will further eliminate the gauge freedom in these numerators. On the other hand, when 1 and $n$ are a different species from the rest, crossing symmetry involving them is necessarily absent.
} 
On the other hand, CHY formalism provides a way to compute local numerators in the DDM basis through eq. (1.1). Although the possibility was recognized shortly after the discovery of CHY [26], the explicit construction was highly nontrivial and came much later. ${ }^{3}$ The computation for up to three gluons and two traces in Yang-Mills-scalar (YMS) theory [57] was done in [58-61]. Shortly after, a recursive expansion for the singletrace sector of YMS was proposed based on gauge invariance [62] and a direct ansatz construction [63], which was soon proved and refined by expanding the CHY integrand recursively [64]. Follow-up works further extended the algorithm to pure Yang-Mills [65] and multi-trace sector [66]. A crucial feature common to all these constructions is that the DDM basis numerators are given by assigning kinematic factors to spanning trees on $n$ vertices - connected graphs on $n$ vertices with no cycles.

While the spanning tree construction is generic, the need for a reference ordering (RO) causes the numerators to be a function of more than just the particle ordering, and thus not manifest crossing symmetry,

$$
N_{\mathrm{RO}}(1, \beta, n) \neq\left. N_{\mathrm{RO}}(1,2, \ldots, n)\right|_{2 \rightarrow \beta_{2}, 3 \rightarrow \beta_{3}, \ldots, n-1 \rightarrow \beta_{n-1}} .
$$

Although this is not in line with eq. (1.4), the full amplitude remains permutation invariant due to the nontrivial kernel of $m(1, \alpha, n \mid 1, \beta, n)$, i.e. the BCJ amplitude relations. Crossing symmetry can be recovered by averaging over all the ROs (RO-average). However, this prescription introduces a factorial computational complexity, which is highly undesired. We note that the mathematical origin of this $\mathrm{RO}$ dependence roots in the Laplace expansion of determinants and Pfaffians: when one performs the expansion along a chosen row, the manifest exchange symmetry between this row and the rest is broken. Different choices lead to different patterns of breaking the symmetry. Alternatively, the prescriptions presented in $[62,64-66]$ can be derived from conventional string theory $[32,34,61]$ and differential operators [67]. The stringy interpretation of the RO is a priority list for breaking the subcycles formed by world-sheet variables using integration-by-parts relations.

In section 2 and part of section 3, we will review the RO-based construction of sYM and multi-trace YMS numerators. While the technique summarized in algorithm 1 is mostly equivalent to those in refs. [34, 62, 64-66], we streamline the algorithm with new notations that are more suitable to describe the novel crossing symmetric contruction discussed later. In addition, we propose a new baseline expansion (2.16) for YMS that works for any choice of particle 1 and $n$ (gluon, scalars in the same or different traces). ${ }^{4}$

While the lack of crossing symmetry does not pose a problem when studying tree-level amplitudes, it can be challenging to deal with when using trees as part of loop calculations. The inability to relabel numerators prevents systematic approaches using graph isomorphisms that might generalize to loops. However, the CHY formalism has been extended to handle one-loop directly. This representation of loop amplitudes can be derived from ambitwistor string models based on a nodal Riemann sphere [37, 68-70], which in turn allows direct calculation from an $(n+2)$-point tree-level amplitude through a forward limit [71, 72].

\footnotetext{
${ }^{3}$ See ref. [56] for a generic construction of non-local BCJ numerators using CHY integration rules.

${ }^{4} \mathrm{It}$ is a natural generalization of ref. [66], where 1 and $n$ are fixed to be scalars.
} 
Following this approach, one can avoid the difficulty of dealing with contributions from different spin structures at one-loop level. The resultant loop integrands have the prominent feature that they contain linearized propagators rather than the standard Feynman-type ones. Moreover, kinematic numerators satisfying BCJ relations will automatically lead to such numerators at one-loop, which can be directly fed into a double-copy construction for gravity theories $[73,74]$. These method of obtaining BCJ-compatible one-loop numerators have been explored by various works in literature [73-76].

Thus, high multiplicity loops in many theories are accessible from high multiplicity trees via $\mathrm{CHY}$ and forward limits. The first technical difficulty to overcome towards this goal is the need to efficiently produce tree-level BCJ numerators with very high multiplicity. As the main goal of this paper, we develop an improved algorithm to arrive at crossing symmetric numerators without performing the explicit after-the-fact RO-average. In other words, the new method gets rid of RO-dependence completely. The procedure, presented in algorithm 2, works by processing the structure of a spanning tree recursively rather than sequentially over arbitrarily split paths. The recursive structure allows kinematic weights to be assigned at each node which locally incorporate the appropriate tensor structures and normalizations such that a spanning tree processed in the new algorithm gives the same result as performing the RO-average after the old construction [34, 62, 6466]. The bulk of this paper is dedicated to examining the combinatorics and kinematic factors needed at each stage of the recursion. In section 3.2, we derive the prescription for sYM amplitudes containing at most two fermions. ${ }^{5}$ We then generalize it to multi-trace sector of YMS theory in section 3.4. Our algorithm will automatically respect the crossing symmetry among gluons and scalar traces, all of which are not manifest when a RO is required. We have checked that our algorithm can provide complete ten-point results within minutes on a laptop. In a forthcoming paper [78], we will feed these tree-level numerators into the forward-limit machinery to study one-loop numerators with various amount of supersymmetry.

Additionally, since our formulation is dimensionally agnostic, it is possible to embed the numerators in higher dimensions in such a way as to introduce mass to specific particles via dimensional compactification. In particular, it is straightforward to perform this embedding such that only particles 1 and $n$ obtain a mass. These types of diagrams are related to those needed for the recent 3PM (third post-Minkowski) calculation of ref. [21] as input to the unitarity cuts used for constructing the two-loop classical amplitude.

Our paper is organized as follows. In section 2, we review the general structure of the CHY representation of integrands. This section specifically focuses on the initial expansion of $\mathrm{Pf}^{\prime}$ into pure $\mathrm{Pf}$ weighted by kinematics and Parke-Taylor factors. We cover pure gluon, two-fermion, and multi-trace YMS. Section 3 begins by reviewing the spanning tree method for CHY (half-)integrand construction. It then presents our derivation of a recursive restructuring of the spanning tree calculation which implicitly performs an average over reference ordering, as well as providing some examples of the new technique. Finally, in section 4, we briefly discuss introducing masses to our numerators. Appendix A provides

\footnotetext{
${ }^{5}$ Numerators with more fermions can be obtained from the pure-spinor formalism of sYM [43, 77].
} 
our explicit conventions for the CHY matrices and normalizations. Appendix B describes our MATHEMATICApackage that implements the spanning tree numerator construction.

\section{CHY integrands and baseline expansion}

In the CHY representation, $n$-point tree-level color-ordered amplitudes are expressed as an integral over the moduli space of $n$-punctured Riemann sphere,

$$
A_{n}^{\text {tree }}(\rho)=\mathscr{N} \int d \mu_{n}^{\text {tree }} \operatorname{PT}(\rho) \mathcal{I}_{n}^{\text {tree }}, \quad d \mu_{n}^{\text {tree }} \equiv \frac{d^{n} \sigma}{\operatorname{Vol}[\operatorname{SL}(2, \mathbb{C})]} \prod_{i=1}^{n} \delta\left(E_{i}\right),
$$

where the delta functions in the measure localize the integral to the $(n-3)$ ! solutions of the scattering equations [24-26],

$$
E_{i} \equiv \sum_{\substack{j=1 \\ j \neq i}}^{n} \frac{k_{i} \cdot k_{j}}{\sigma_{i j}}=0, \quad \sigma_{i j} \equiv \sigma_{i}-\sigma_{j} .
$$

The color ordering is encoded in the Parke-Taylor factor $\operatorname{PT}(\rho)$, which is a weight-two function under the $\mathrm{SL}(2, \mathbb{C})$ transformation $\sigma_{i} \rightarrow \frac{a \sigma_{i}+b}{c \sigma_{i}+d}$ with $a d-b c=1$. The (half-)integrand $\mathcal{I}_{n}^{\text {tree }}$ depends on the kinematic data (momentum and polarization). It is constructed as an $\mathrm{SL}(2, \mathbb{C})$ weight-two function as well such that the full integration form is $\mathrm{SL}(2, \mathbb{C})$ invariant. The $\mathrm{SL}(2, \mathbb{C})$ gauge redundancy, combined with the $\frac{1}{\operatorname{Vol}[\mathrm{SL}(2, \mathbb{C})]}$, can be removed by fixing the positions of three punctures and dropping three redundant scattering equations. For example, we can choose

$$
d \mu_{n}^{\text {tree }}=\left(\sigma_{1, n-1} \sigma_{n-1, n} \sigma_{n 1}\right)^{2} \prod_{i=2}^{n-2}\left[d \sigma_{i} \delta\left(E_{i}\right)\right],
$$

where $\left(\sigma_{1}, \sigma_{n-1}, \sigma_{n}\right)=(0,1, \infty)$. The normalization $\mathscr{N}$ contains the coupling constants of the theory, which we match against the explicit Lagrangian in section A.

\subsection{Super-Yang-Mills (sYM) theories}

We first focus on sYM theories. The CHY integrand for pure gluon amplitudes is [25]

$$
\mathcal{I}_{n}^{\text {gluon }}=\operatorname{Pf}^{\prime}(\Psi) .
$$

The full definition of the reduced Pfaffian $\operatorname{Pf}^{\prime}(\Psi)$ is given in section A. One can rewrite $\operatorname{Pf}^{\prime}(\Psi)$ as a baseline expansion on the support of the scattering equations [62, 65, 79],

$$
\mathcal{I}_{n}^{\text {gluon }} \stackrel{\mathrm{SE}}{=} \sum_{(G, B) \in \text { Part }_{2}(2,3, \ldots, n-1)} \operatorname{Pf}\left(\Psi_{G}\right) \sum_{\rho \in S_{|B|}} \operatorname{PT}(1, \rho, n) W_{\text {gluon }}(1, \rho, n),
$$

where the first summation is over all the bi-partitions of the set $\{2,3, \ldots, n-1\}$, and the second summation is over all the permutations of the set $B .{ }^{6}$ The matrix $\Psi_{G}$ is a submatrix

\footnotetext{
${ }^{6}$ This sum is often written in the literature as over $\{2, \ldots, n-1\}=A \cup B$, but we wish to avoid abuse of notation since $A$ and $B$ are required to be disjoint.
} 
of $\Psi$ in which the rows and columns are restricted to the set $G$ (see section A for more details). We note that both $G$ and $B$ can be empty. In particular, when $G=\emptyset, \operatorname{Pf}(\emptyset)=1$. We call $(1, \rho, n)$ a baseline and the baseline factor $W_{\text {gluon }}$ is given by

$$
W_{\text {gluon }}(1, \rho, n)=(-1)^{|\rho|} \epsilon_{1} \cdot f_{\rho_{1}} \cdot f_{\rho_{2}} \ldots \cdot f_{\rho_{|B|}} \cdot \epsilon_{n}
$$

where $f_{i}^{\mu \nu} \equiv k_{i}^{\mu} \epsilon_{i}^{\nu}-\epsilon_{i}^{\mu} k_{i}^{\nu}$ is the linearized field strength for gluon $i$. Our nomenclature is inspired by the spanning tree expansion scheme for $\mathrm{Pf}^{\prime}$, which will be discussed in section 3. For the derivation of eq. (2.5), we refer the interested readers to the aforementioned references. From the ambitwistor string point of view, singling out gluon 1 and $n$ amounts to assigning ghost picture -1 to the vertex operators in the RNS formalism [35]. The gauge invariance of these two particles are not manifest, but can be recovered by using the scattering equations.

Interestingly, the CHY integrand with exactly two external fermions takes a similar baseline expansion form. If we fix the fermions to be particle 1 and $n$, the only difference from eq. (2.5) is in the baseline factor [78],

$$
\mathcal{I}_{n}^{2 \mathrm{f}}\left(1_{\mathrm{f}}, 2, \ldots, n-1, n_{\mathrm{f}}\right)=\sum_{(G, B) \in \text { Part }_{2}(2,3, \ldots, n-1)} \operatorname{Pf}\left(\Psi_{G}\right) \sum_{\rho \in S_{|B|}} \operatorname{PT}(1, \rho, n) W_{2 \mathrm{f}}\left(1_{\mathrm{f}}, \rho, n_{\mathrm{f}}\right) .
$$

There exist two forms of the baseline factor $W_{2 \mathrm{f}}$, equivalent on the support of the scattering equations and gauge transformations. In $D=10$, they are given $\mathrm{as}^{7}$

$$
\begin{aligned}
& W_{2 \mathrm{f}}^{(1)}\left(1_{\mathrm{f}}, \rho, n_{\mathrm{f}}\right)=\left\{\begin{array}{ll}
\frac{(-1)^{|\rho|}}{2}\left(\chi_{1} f_{\rho_{1}} \ldots f_{\rho_{i-1}} \notin_{\ell} f_{\rho_{i+1}} \ldots f_{\rho_{|B|}} \chi_{n}\right) & \rho_{i}=\ell \\
0 & \ell \notin \rho
\end{array},\right. \\
& W_{2 \mathrm{f}}^{(2)}\left(1_{\mathrm{f}}, \rho, n_{\mathrm{f}}\right)=(-1)^{|\rho|}\left(\chi_{1} f_{\rho_{1}} f_{\rho_{2}} \ldots f_{\rho_{|B|}} \xi_{n}\right),
\end{aligned}
$$

where in the first equation, $\ell$ is an arbitrary gluon and the baseline factor vanishes if $\ell \notin B$. Eq. (2.8b) has the benefit of being manifestly crossing symmetric and gauge invariant in all of the gluons. In both expressions, the gamma-matrix convention is $\left\{\gamma^{\mu}, \gamma^{\nu}\right\}=2 \eta^{\mu \nu}$, and

$$
\notin_{\ell} \equiv \epsilon_{\ell}^{\mu} \gamma_{\mu}, \quad f_{i} \equiv \frac{1}{8} f_{i}^{\mu \nu}\left[\gamma_{\mu}, \gamma_{\nu}\right]=\frac{1}{2} \not k_{i} \notin_{i} .
$$

The Weyl-Majorana spinor $\chi_{i}$ is the solution to the equation of motion $\not k_{i} \chi_{i}=0$, and the spinor $\xi_{i}$ is related to $\chi_{i}$ through $k_{i} \xi_{i}=\chi_{i}$. The expressions for $D<10$ can be obtained through a dimensional reduction. In particular, for $D=4$ we can use

$$
\chi_{i} \rightarrow\left\langlei | ^ { \alpha , I } \oplus \left[\left. i\right|_{\dot{\alpha}, I}, \quad \xi_{i} \rightarrow \frac{|q\rangle_{\alpha, I}}{\langle i q\rangle} \oplus \frac{\mid q]^{\dot{\alpha}, I}}{[i q]}\right.\right.
$$

\footnotetext{
${ }^{7}$ The two expressions originate from the two ways to assign ghost picture to the vertex operators in the RNS formalism of ambitwistor string: eq. (2.8a) comes from assigning ghost picture $-1 / 2$ to both fermion vertex operators and ghost picture -1 to the gluon vertex operator $m$, while eq. (2.8b) comes from assigning ghost picture $-1 / 2$ and $-3 / 2$ to the fermion vertex operators and ghost picture zero to all the gluons. We have found the two expressions useful for studying different aspects of forward limits, see ref. [78] for details.
} 
where $\alpha, \dot{\alpha}=1,2$ are left- and right-handed Weyl spinor indices, $I=1,2,3,4$ is the $\mathrm{SU}(4)$ R-symmetry index and the $\oplus$ is a formal sum over the representation spaces. In $D=6$, we can similarly construct $\chi$ and $\xi$ using $6 \mathrm{D}$ spinor helicity variables [80]

$$
\left.\chi_{i} \rightarrow\left|i_{a}\right\rangle^{A} \oplus \mid i_{\dot{a}}\right]_{A}, \quad \xi_{i} \rightarrow \frac{\left.\mid q_{\dot{b}}\right]_{A}\left[q^{\dot{b}} i_{a}\right\rangle}{2 q \cdot k_{i}} \oplus \frac{\left|q^{b}\right\rangle^{A}\left\langle q_{b} i_{\dot{a}}\right]}{2 q \cdot k_{i}}
$$

where the spinors $|i\rangle^{A}$ and $\left.\mid i\right]_{A}$ are in the fundamental and anti-fundamental representation of $\mathrm{SU}(4)$, and $a, \dot{a}=1,2$ are the $\mathrm{SU}(2) \times \mathrm{SU}(2)$ little group indices. In both eqs. (2.10) and (2.11), $q$ is a reference vector that obeys $q \cdot k_{i} \neq 0$ but otherwise is arbitrary. The final amplitudes do not depend on the choice of $q$.

The baseline expansion brings the pure gluon and two-fermion CHY integrand into a unified form. In order to obtain the DDM basis numerators $N(1, \beta, n)$, we need a systematic way to expand $\operatorname{Pf}\left(\Psi_{G}\right)$. In section 3 , we will discuss how this can be done algorithmically using spanning trees.

\subsection{Yang-Mills-scalar}

Both eqs. (2.5) and (2.7) can be interpreted as expanding the pure-gluon/two-fermion integrand in terms of those for single-trace YMS amplitudes. If $G$ is the set of gluons and $(1, \tau, n)$ is the (second) color ordering of the bi-adjoint scalars, we can write the halfintegrand as [27]

$$
\mathcal{I}_{n}^{\mathrm{YMS}}((1, \tau, n) \mid G)=\operatorname{PT}(1, \tau, n) \operatorname{Pf}\left(\Psi_{G}\right)
$$

which corresponds to choosing the baseline factor to be $W(1, \tau, n)=\delta_{\tau, \rho}$ in eq. (2.5).

In fact, there exists a similar baseline expansion for the multi-trace YMS integrand [28],

$$
\mathcal{I}_{n}^{\mathrm{YMS}}\left(\tau_{1}, \tau_{2}, \ldots, \tau_{m} \mid G\right)=\mathrm{PT}\left(\tau_{1}\right) \operatorname{PT}\left(\tau_{2}\right) \ldots \mathrm{PT}\left(\tau_{m}\right) \mathrm{Pf}^{\prime} \Pi\left(\tau_{1}, \tau_{2}, \ldots, \tau_{m} \mid G\right),
$$

where $G$ denotes the set of gluons and $\left\{\tau_{1}, \ldots, \tau_{m}\right\}$ the scalar color traces. Since in many of our future manipulations, scalar traces are treated as a single object and have similar behavior as a gluon, we thus define for convenience the set

$$
\left\{\mathrm{t}_{1}, \mathrm{t}_{2}, \ldots, \mathrm{t}_{\mathrm{N}}\right\}=\left\{\tau_{1}, \tau_{2}, \ldots, \tau_{m}\right\} \cup G, \quad \mathrm{~N}=m+|G|,
$$

where each entry $t_{i}$ is either a scalar trace or a single gluon. The matrix $\Pi$ is $2 \mathrm{~N}$ dimensional, in which the entries are labeled by gluons and scalar traces. The explicit form of $\Pi$ is given in section A. When $m=0$ and 1 , we have

$$
\operatorname{Pf}^{\prime} \Pi\left(\tau_{1} \mid G\right)=\operatorname{Pf}\left(\Psi_{G}\right), \quad \quad \operatorname{Pf}^{\prime} \Pi(\emptyset \mid G)=\operatorname{Pf}^{\prime}(\Psi)
$$

such that eq. (2.13) reduces to the single trace and pure gluon integrand respectively.

For the multitrace integrand eq. (2.13), the baseline expansion involves integrands with total number of gluons and trace reduced. It applies when the end points of the baseline, 
chosen as 1 and $n$, do not belong to the same scalar trace. We can schematically write the expansion of $\mathcal{I}_{n}^{\mathrm{YMS}}\left(\tau_{1}, \tau_{2}, \ldots, \tau_{m} \mid G\right)=\mathcal{I}_{n}^{\mathrm{YMS}}\left(\mathrm{t}_{1}, \mathrm{t}_{2}, \ldots, \mathrm{t}_{\mathrm{N}}\right)$ as

$$
\begin{aligned}
\mathcal{I}_{n}^{\mathrm{YMS}}\left(\mathrm{t}_{1}, \mathrm{t}_{2}, \ldots, \mathrm{t}_{\mathrm{N}}\right) \stackrel{\mathrm{SE}}{=} & \sum_{(A, B) \in \mathbf{P a r t}_{2}\left(\mathrm{t}_{2}, \ldots, \mathrm{t}_{\mathrm{N}-1}\right)} \operatorname{Pf}^{\prime} \Pi(\left\{\mathrm{t}_{1}, B, \mathrm{t}_{\mathrm{N}}\right\}, \overbrace{\alpha_{1}, \ldots, \alpha_{s} \mid G^{\prime}}^{\prime}) \\
& \times \operatorname{PT}\left(\alpha_{1}\right) \ldots \mathrm{PT}\left(\alpha_{s}\right) \sum_{\rho \in S_{\left\{\mathrm{t}_{1}, B, \mathrm{t}_{\mathrm{N}}\right\} \backslash\{1, n\}}}^{A} \operatorname{PT}(1, \rho, n) W_{\mathrm{MT}}(1, \rho, n),
\end{aligned}
$$

where we assume that $1 \in \mathrm{t}_{1}$ and $n \in \mathrm{t}_{\mathrm{N}}$. At this point, each term in the summation of eq. (2.16) is a multi-trace integrand with 1 and $n$ in the same trace $(1, \rho, n)$, where $\rho$ is a permutations of $\left\{\mathrm{t}_{1}, B, \mathrm{t}_{\mathrm{N}}\right\}$ with $1 \in \mathrm{t}_{1}$ and $n \in \mathrm{t}_{\mathrm{N}}$ fixed at the ends. Meanwhile, the set $A$ is given by

$$
A=\left\{\mathrm{t}_{a_{1}}, \ldots, \mathrm{t}_{a_{|A|}}\right\}=\left\{\alpha_{1}, \ldots, \alpha_{s}\right\} \cup G^{\prime}
$$

where $\alpha_{i}$ 's are scalar traces and $G^{\prime}$ a subset of gluons. In section 3.4, we will discuss the spanning tree algorithm to evaluate such integrands. Before moving into more details, we note that eq. (2.16) was first derived for pure scalar integrands in ref. [66]. However, the most generic form as given here is new. We have explicitly checked it for varoius gluon and trace configurations against direct numeric evaluations of eq. (2.1) up to very high multiplicities. It can be proved in a similar manner as appendix $\mathrm{C}$ and $\mathrm{D}$ of ref. [66], but we omit such a proof here for conciseness.

We now introduce some essential tools to specify the baseline factor $W_{\mathrm{MT}}(1, \rho, n)$ in the second row of eq. (2.16). Treating each scalar trace in $\left\{\mathrm{t}_{1}, B, \mathrm{t}_{\mathrm{N}}\right\}$ as a single object, we can define a sub-ordering $\left(a_{i}, \omega_{i}, b_{i}\right)$ for each $\mathrm{t}_{i} \in\left\{\mathrm{t}_{1}, B, \mathrm{t}_{\mathrm{N}}\right\}$ by simply restricting the baseline $(1, \rho, n)$ to the elements of $\mathrm{t}_{i}$,

$$
\left.\left(a_{i}, \omega_{i}, b_{i}\right) \equiv(1, \rho, n)\right|_{\mathrm{t}_{i}},
$$

where $a_{i}$ and $b_{i}$ are respectively the first and last element of $\mathrm{t}_{i}$ that appear in the baseline. If $\mathrm{t}_{i}$ is a scalar trace, then $\left(a_{i}, \omega_{i}, b_{i}\right)$ is a permutation of $\mathrm{t}_{i}$, while for $\mathrm{t}_{i}$ being a gluon, we simply define $a_{i}=b_{i}=\mathrm{t}_{i}$ and $\omega_{i}=\emptyset$. Given such a pair $\left(a_{i}, b_{i}\right) \subset \mathrm{t}_{i}$, we define the collection of Kleiss-Kuijf-compatible (KK-compatible) permutations as

$$
\mathrm{KK}\left[\mathrm{t}_{i}, a_{i}, b_{i}\right]=\left\{\begin{array}{ll}
\left\{\left(a_{i}, \omega_{i}, b_{i}\right) \mid \omega_{i} \in X_{i} ш Y_{i}^{T}\right\} & \text { if } \mathrm{t}_{i}=\left(a_{i}, X_{i}, b_{i}, Y_{i}\right) \text { is a trace } \\
\left(\mathrm{t}_{i}\right) & \text { if } \mathrm{t}_{i} \text { is a gluon }
\end{array},\right.
$$

where the set $X_{i}$ and $Y_{i}$ can be obtained by cyclically rotating $a_{i}$ to the first element. They are exactly the orderings generated by a Kleiss-Kuijf relation [81], and hence the name. The sign generated by this operation

$$
\mathfrak{s g n}_{a_{i}, \omega_{i}, b_{i}}^{\mathrm{t}_{i}} \equiv \begin{cases}(-1)^{\left|Y_{i}\right|} & \text { if }\left(a_{i}, \omega_{i}, b_{i}\right) \in \mathrm{KK}\left[\mathrm{t}_{i}, a_{i}, b_{i}\right] \\ 0 & \text { otherwise }\end{cases}
$$


will be included in the baseline factor $W_{\mathrm{MT}}(1, \rho, n)$. Note that for a gluon, we always have $\mathfrak{s g n}_{a_{i}, b_{i}}^{\mathrm{t}_{i}}=\mathfrak{s g n} \mathfrak{t}_{\mathrm{t}_{i}, \mathrm{t}_{i}}^{\mathrm{t}_{i}}=1$ since $\left|Y_{i}\right|=0$ by definition. This function is nonzero only for KK-compatible permutations of $t_{i}$.

In addition, we define the ordering function $\mathscr{O}$ that rearranges the baseline $(1, \rho, n)$ by comparing the first element of the $|B|+2$ sub-orderings $\left(a_{i}, \omega_{i}, b_{i}\right)$ : the triplet $\left(a_{i}, \omega_{i}, b_{i}\right)$ precedes $\left(a_{j}, \omega_{j}, b_{j}\right)$ if $a_{i}$ precedes $a_{j}$ in $(1, \rho, n)$, namely,

$$
\mathscr{O}_{(1, \rho, n)}=(\overbrace{a_{0}, \omega_{0}, b_{0}}^{\lambda_{0}}, \overbrace{a_{1}, \omega_{1}, b_{1}}^{\lambda_{1}}, \ldots, \overbrace{a_{|B|}, \omega_{|B|}, b_{|B|}}^{\lambda_{|B|}}, \overbrace{a_{|B|+1}, \omega_{|B|+1}, b_{|B|+1}}^{\lambda_{|B|+1}} .
$$

We can also define the coarse-grained ordering function

$$
\mathscr{O}_{(1, \rho, n)}^{\mathrm{cg}}=\left(\lambda_{0}, \lambda_{1}, \ldots, \lambda_{|B|}, \lambda_{|B|+1}\right)
$$

that extracts a permutation formed by the $|B|+2$ elements in $\left\{\mathrm{t}_{1}, B, \mathrm{t}_{\mathrm{N}}\right\}$, which will be useful later in section section 3.4. In fact, we must have $\lambda_{0}=\mathrm{t}_{1}$ and $a_{0}=1$ by construction. In particular, we will be interested in those baselines that are $\mathscr{O}$-invariant,

$$
(1, \rho, n)=\mathscr{O}_{(1, \rho, n)} .
$$

For these cases, we must also have $\lambda_{|B|+1}=\mathrm{t}_{\mathrm{N}}$ and $b_{|B|+1}=n$. In other words, in $\mathscr{O}_{-}$ invariant baselines every scalar trace is consecutive in the sense that between $a_{i}$ and $b_{i}$ there are no elements of other traces or gluons. To characterize these permutations, we introduces a new object

$$
\mathcal{B}_{(1, \rho, n)}\left(\lambda_{i} \mid \lambda_{j}\right)=\left\{x \in \lambda_{i} \mid x \text { before } a_{j} \text { in }(1, \rho, n)\right\},
$$

that measures the "mixing level" between $\left(a_{i}, \omega_{i}, b_{i}\right)$ and $\left(a_{j}, \omega_{j}, b_{j}\right)$,

$$
\mathcal{B}_{(1, \rho, n)}\left(\lambda_{i} \mid \lambda_{j}\right)= \begin{cases}\lambda_{i} & \text { all of } \lambda_{i} \text { come before } a_{j} \text { in }(1, \rho, n) \\ \tilde{\lambda}_{i} \subsetneq \lambda_{i} & \text { otherwise }\end{cases}
$$

where $\tilde{\lambda}_{i}$ is a proper subset of $\lambda_{i}$ and it can be empty. Thus the $\mathscr{O}$-invariant baselines have the form

$$
(\overbrace{1, \theta_{1}, j_{1}}^{t_{1}}, \overbrace{a_{1}, \omega_{1}, b_{1}}^{\lambda_{1}}, \overbrace{a_{2}, \omega_{2}, b_{2}}^{\lambda_{2}}, \ldots, \overbrace{a_{|B|}, \omega_{|B|}, b_{|B|},}^{\lambda_{|B|}} \overbrace{i_{\mathrm{N}}, \theta_{\mathrm{N}}, n}^{t_{\mathrm{N}}})
$$

and are characterized by $\mathcal{B}_{(1, \rho, n)}\left(\lambda_{i} \mid \lambda_{i+1}\right)=\lambda_{i}$ for $\lambda_{i} \in\left\{\lambda_{0}, \lambda_{1}, \ldots, \lambda_{|B|}\right\}$. We must have $t_{\mathrm{N}}$ as the last consecutive block because $n \in \mathrm{t}_{\mathrm{N}}$ is the endpoint of the baseline. If there exists a $\lambda_{j}$ after $\mathrm{t}_{\mathrm{N}}$, then we must have $\mathcal{B}_{(1, \rho, n)}\left(\mathrm{t}_{\mathrm{N}} \mid \lambda_{j}\right) \subsetneq \mathrm{t}_{\mathrm{N}}$ and thus the $\mathscr{O}$-invariant condition is violated.

With the above preparations, we now reach the punchline of the multi-trace baseline expansion: the baseline factor $W_{\mathrm{MT}}(1, \rho, n)$ is nonzero if and only if

- the baseline $(1, \rho, n)$ is $\mathscr{O}$-invariant: $(1, \rho, n)=\mathscr{O}_{(1, \rho, n)}$;

- the restriction of $(1, \rho, n)$ to every $\lambda_{i} \in\left\{\mathrm{t}_{1}, B, \mathrm{t}_{\mathrm{N}}\right\}$, denoted as $\left(a_{i}, \omega_{i}, b_{i}\right)$, is KKcompatible. 
For such permutations, $W_{\mathrm{MT}}(1, \rho, n)=W_{\mathrm{MT}}\left(\mathscr{O}_{(1, \rho, n)}\right)$ is given by

$$
\begin{aligned}
& W_{\mathrm{MT}}(1, \rho, n)=W_{\mathrm{MT}}(\overbrace{1, \theta_{1}, j_{1}}^{\mathrm{t}_{1}}, \overbrace{a_{1}, \omega_{1}, b_{1}}^{\lambda_{1}}, \overbrace{a_{2}, \omega_{2}, b_{2}}^{\lambda_{2}}, \ldots, \overbrace{a_{|B|}, \omega_{|B|}, b_{|B|}}^{\lambda_{|B|}} \overbrace{i_{\mathrm{N}}, \theta_{\mathrm{N}}, n}^{t_{\mathrm{N}}}) \\
& =(-1)^{\left|B_{g}\right|} \mathcal{E}_{1, \theta_{1}, j_{1}}^{\mathrm{t}_{1}} \cdot \mathcal{T}_{a_{1}, \omega_{1}, b_{1}}^{\lambda_{1}} \cdot \mathcal{T}_{a_{2}, \omega_{2}, b_{2}}^{\lambda_{2}} \cdots \cdot \mathcal{T}_{a_{|B|}, \omega_{|B|}, b_{|B|}}^{\lambda_{|B|}} \cdot \widetilde{\mathcal{E}}_{i_{\mathrm{N}}, \theta_{\mathrm{N}}, n}^{\mathrm{t}_{\mathrm{N}}} \cdot
\end{aligned}
$$

where $\left|B_{g}\right|$ is the number of gluons on the baseline, and

$$
\begin{aligned}
& \left(\mathcal{T}_{a_{i}, \omega_{i}, b_{i}}^{\lambda_{i}}\right)^{\mu \nu}=\left\{\begin{array}{cc}
\mathfrak{s g n}_{a_{i}, \omega_{i}, b_{i}}^{\lambda_{i}}\left(-k_{a_{i}}^{\mu} k_{b_{i}}^{\nu}\right) & \lambda_{i} \text { is a trace and } \mathcal{B}_{(1, \rho, n)}\left(\lambda_{i} \mid \lambda_{i+1}\right)=\lambda_{i} \\
0 & \lambda_{i} \text { is a trace and } \mathcal{B}_{(1, \rho, n)}\left(\lambda_{i} \mid \lambda_{i+1}\right) \subsetneq \lambda_{i} \\
f_{\lambda_{i}}^{\mu \nu} & \lambda_{i} \text { is a gluon }
\end{array}\right. \\
& \left(\mathcal{E}_{a_{i}, \omega_{i}, b_{i}}^{\lambda_{i}}\right)^{\mu}=\left\{\begin{array}{cc}
\mathfrak{s g n}_{a_{i}, \omega_{i}, b_{i}}^{\lambda_{i}}\left(-k_{b_{i}}^{\mu}\right) & \lambda_{i} \text { is a trace } \\
\epsilon_{\lambda_{i}}^{\mu} & \lambda_{i} \text { is a gluon }
\end{array}\right)_{\left(\widetilde{\mathcal{E}}_{a_{i}, \omega_{i}, b_{i}}^{\lambda_{i}}\right)^{\mu}}^{\lambda^{\mu}}=\left\{\begin{array}{cc}
\mathfrak{s g n}_{a_{i}, \omega_{i}, b_{i}}^{\lambda_{i}} k_{a_{i}}^{\mu} & \lambda_{i} \text { is a trace } \\
\epsilon_{\lambda_{i}}^{\mu} & \lambda_{i} \text { is a gluon }
\end{array}\right.
\end{aligned}
$$

Thus, the baseline factor for a generic permutation can be written as

$$
W_{\mathrm{MT}}(1, \rho, n)=(-1)^{\left|B_{g}\right|} \mathcal{E}_{a_{0}, \omega_{0}, b_{0}}^{\lambda_{0}} \cdot \mathcal{T}_{a_{1}, \omega_{1}, b_{1}}^{\lambda_{1}} \cdot \mathcal{T}_{a_{2}, \omega_{2}, b_{2}}^{\lambda_{2}} \cdot \ldots \cdot \mathcal{T}_{a_{|B|}, \omega_{|B|}, b_{|B|}}^{\lambda_{|B|}} \cdot \widetilde{\mathcal{E}}_{a_{|B|+1}, \omega_{|B|+1}, b_{|B|+1}}^{\lambda_{|B|+1}},
$$

where $\left(\lambda_{0}, \lambda_{1}, \ldots, \lambda_{|B|}, \lambda_{|B|+1}\right)=\mathscr{O}_{(1, \rho, n)}^{\text {cg }}$ is the coarse-grained baseline. The $\mathcal{B}_{(1, \rho, n)}$ and $\mathfrak{s g n}$ in eq. (2.28) ensure any permutations that are not of the form of eq. (2.27) are immediately set to 0 . With the help of this zeroing, the summation in the second line of eq. (2.16) automatically reduces to

$$
\sum_{\rho \in S_{\left\{\mathrm{t}_{1}, B, \mathrm{t}_{\mathrm{N}}\right\} \backslash\{1, n\}}} \mathrm{PT}(1, \rho, n) W_{\mathrm{MT}}(1, \rho, n)=\sum_{\substack{\text { O-invariant } \\ \text { KK-compatible }}} \mathrm{PT}(1, \rho, n) W_{\mathrm{MT}}(1, \rho, n) .
$$

Finally, we remark on the case that both the baseline endpoints 1 and $n$ belong to the same trace, say $\tau_{1}$, which is left out by eq. (2.16). Starting from eq. (2.13), we simply write

$$
\begin{aligned}
\mathcal{I}_{n}^{\mathrm{YMS}}\left(\tau_{1}, \tau_{2}, \ldots, \tau_{m} \mid G\right)= & \operatorname{PT}\left(\tau_{2}\right) \ldots \operatorname{PT}\left(\tau_{m}\right) \operatorname{Pf}^{\prime} \Pi\left(\tau_{1}, \tau_{2}, \ldots, \tau_{m} \mid G\right) \\
& \times \sum_{\rho \in \mathrm{KK}\left[\tau_{1}, 1, n\right]} \operatorname{PT}(1, \rho, n) W_{\mathrm{MT}}(1, \rho, n)
\end{aligned}
$$

by using Kleiss-Kuijf relations. Here, we can naturally reduce the definition of $W_{\mathrm{MT}}$ given in eq. (2.27) to the single-trace baseline,

$$
W_{\mathrm{MT}}(\overbrace{1, \rho, n}^{\tau_{1}})=W_{\mathrm{MT}}\left(\mathscr{O}_{(1, \rho, n)}\right)=\mathfrak{s g n}_{1, \rho, n}^{\tau_{1}} .
$$

We note that since the $\mathfrak{s g n}$ function is only nonzero for KK-compatible baselines, we can trivially extend the summation range in eq. (2.30) to $S_{\tau_{1} \backslash\{1, n\}}$. Although this rewriting does not reduce the total number of scalar traces and gluons, it puts the CHY integrand into the same form as eq. (2.16), from which the half-ladder numerators can be obtained through a unified spanning-tree algorithm given in section 3.4. We note that the expansion (2.30) is first proved at the amplitude level in ref. [66]. 


\section{DDM basis numerators from CHY integrands}

The baseline expansion is the first step of our systematic approach to obtain the DDM basis numerators $N(1, \beta, n)$ from CHY integrands. It arranges the sYM and YMS integrands into an expansion of YMS integrands in which the total number of gluons and traces is reduced. Our next step is to further expand these YMS integrands in terms of the DDM basis ParkeTaylor factors. This is realized through a spanning tree expansion, originally developed in refs. [62, 64, 65, 82] using reference orderings. We review the original formulation before deriving a new approach to the construction.

\subsection{Spanning trees and reference orderings}

We first focus on eqs. (2.5) and (2.7), the baseline expansion for the pure gluon and two-fermion integrand, both of which are a linear combination of single trace integrands $\operatorname{PT}(1, \rho, n) \operatorname{Pf}\left(\Psi_{G}\right)$. Using a different type of recursive expansion, we can write them in terms of the single trace integrands involving fewer gluons [62, 64],

$$
\operatorname{PT}(1, \rho, n) \operatorname{Pf}\left(\Psi_{G}\right) \stackrel{\mathrm{SE}}{=} \sum_{(A, B) \in \text { Part }_{2}(G \backslash l)} \operatorname{Pf}\left(\Psi_{A}\right) \sum_{\alpha \in S_{B}} \sum_{i=0}^{|\rho|} \mathcal{P}\left(l, \alpha, \rho_{i}\right) \mathcal{C}_{(1, \rho, n)}\left(\rho_{i}, \alpha^{T}, l\right) .
$$

On the right hand side, we pick an arbitrary gluon $l \in G$ and sum over all the bi-partitions of the rest. As before, both the set $A$ and $B$ can be empty. We call $\mathcal{P}\left(l, \alpha, \rho_{i}\right)$ a path factor starting from $l$ and terminating at $\rho_{i}$ on the baseline (we define $\rho_{0}=1$ ), with the intermediate points a permutation $\alpha$ of the set $B$,

$$
\mathcal{P}\left(l, \alpha, \rho_{i}\right)=\epsilon_{l} \cdot f_{\alpha_{1}} \cdot f_{\alpha_{2}} \cdot \ldots \cdot f_{\alpha_{|B|}} \cdot k_{\rho_{i}} .
$$

The nomenclature will be clear in the following section in which we represent this expansion in terms of spanning trees. Finally, $\mathcal{C}_{(1, \rho, n)}\left(\rho_{i}, \alpha^{T}, l\right)$ is the Cayley function of the path $(1, \rho)$ and $\left(\rho_{i}, \alpha^{T}, l\right)[82]$,

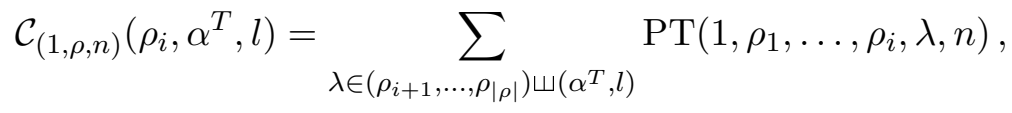

which is a linear combination of Parke-Taylor factors of length $n-|A|$. The final result of this recursive expansion is the Cayley functions of all the $(n-1)$-point spanning trees with a common path $(1, \rho)$, each of which is a linear combination of DDM basis Parke-Taylor factors [82]. It is important to note that in eq. (3.1) the gauge invariance and the crossing symmetry involving the leg $l$ is not manifest. Since one needs to make such choices at each step of the recursion, the final result in general will not display any explicit gauge invariance or crossing symmetry.

With the help of eq. (3.1), one can further expand eqs. (2.5) and (2.7) by Parke-Taylor factors in the DDM basis,

$$
\begin{aligned}
\mathcal{I}_{n}^{\text {gluon } / 2 \mathrm{f}} & \stackrel{\mathrm{SE}}{=} \sum_{(G, B) \in \text { Part }_{2}(2,3, \ldots, n-1)} \operatorname{Pf}\left(\Psi_{G}\right) \sum_{\rho \in S_{|B|}} \operatorname{PT}(1, \rho, n) W_{\text {gluon } / 2 \mathrm{f}}(1, \rho, n) \\
& \stackrel{\mathrm{SE}}{=} \sum_{\beta \in S_{n-2}} \operatorname{PT}(1, \beta, n) N_{\text {gluon } / 2 \mathrm{f}}(1, \beta, n),
\end{aligned}
$$




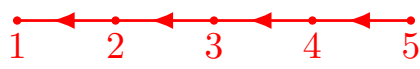

(a)

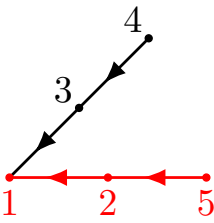

(b)

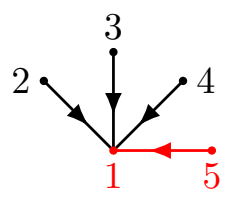

(c)

Figure 1. Examples of trees with various IT memberships: (a) a tree that only belongs to $\operatorname{IT}(1,2,3,4,5),(\mathrm{b})$ a tree that belongs to all $\operatorname{IT}(1,(2) \uplus(3,4), 5),(\mathrm{c})$ a tree that belongs to $\operatorname{IT}(1, \beta, 5)$ for all $\beta \in S_{\{2,3,4\}}$. The baselines are shown in red here and in all future occurances.

where the coefficients $N(1, \beta, n)$ are the DDM basis numerators. They are associated with half-ladder diagrams, and are the master numerators for the BCJ tree relations. A given numerator $N(1, \beta, n)$ can be constructed by assigning kinematic factors to all the $(n-1)$ ! increasing trees consistent with the label ordering [65],

$$
N(1, \beta, n)=\sum_{T \in \mathrm{IT}(1, \beta, n)} N(T),
$$

where the increasing trees are defined as

$$
\operatorname{IT}(1, \beta, n)=\left\{\text { tree } T \mid \begin{array}{c}
\text { any edge } j \rightarrow i \in T \\
\text { satisfies that } i \text { is before } j \text { in }(1, \beta, n)
\end{array}\right\} .
$$

Thus by construction 1 is always the root of $T$ while $n$ is always a leaf. Note that a tree $T$ can belong to several IT $(1, \rho, n)$. Some examples of trees and their IT membership can be seen in figure 1.

Then, for a given increasing tree $T$, the kinematic factor $N(T)$ is evaluated by the following algorithm [65].

Algorithm 1 (Reference Order Evaluation). Construction of the kinematic factor for a spanning tree $T$, using a given reference ordering $R \in S_{n-2}$ :

1. Identify the baseline $(1, \rho, n)$, which is the path $n \rightarrow \rho_{|\rho|} \rightarrow \cdots \rightarrow \rho_{1} \rightarrow 1$ in $T$. Note that $\rho=\emptyset$ is allowed.

2. Split the rest of $T$ into ordered splitting paths $\mathrm{OS}_{R}(T)$ based on a reference ordering $R \in S_{n-2}$ : (a) draw a path from the first element of $R$ towards the baseline, which will either end on the baseline or a previously identified ordered splitting path. (b) move to the next element in $R$ that is not traversed yet, repeat the process until all vertices of $T$ are traversed. For example,

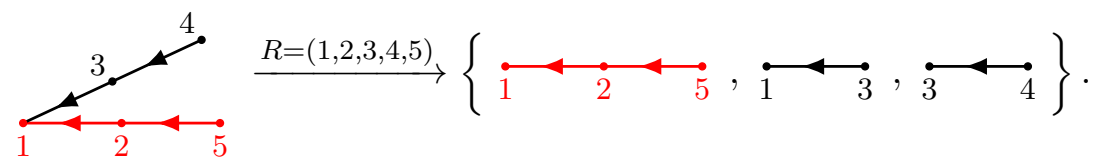

3. Assign kinematic factors to each path

$$
\begin{array}{ll}
\text { baseline } n \rightarrow \rho_{|\rho|} \rightarrow \cdots \rightarrow \rho_{1} \rightarrow 1: & W(1, \rho, n) \\
l \rightarrow \alpha_{1} \rightarrow \cdots \rightarrow \alpha_{|\alpha|} \rightarrow i \in \operatorname{OS}_{R}(T): & \mathcal{P}(l, \alpha, i) .
\end{array}
$$


4. $N(T)$ is the product of all of these paths,

$$
N(T)=W(1, \rho, n) \prod_{(l, \alpha, i) \in \mathrm{OS}_{R}(T)} \mathcal{P}(l, \alpha, i) .
$$

Continuing the example, the ordered splitting in eq. (3.7) leads to

$$
W_{\text {gluon }}(1,2,5) \mathcal{P}(3,1) \mathcal{P}(4,3)=W_{\text {gluon }}(1,2,5)\left(\epsilon_{3} \cdot k_{1}\right)\left(\epsilon_{4} \cdot k_{3}\right) .
$$

The reference ordering $R$ is just a priority list of choosing the special gluon in the recursive expansion (3.1) when multiple choices are present. For simplicity we keep it the same for the evaluation of all the DDM basis numerators. We note that splitting a spanning tree into paths can be viewed as a graphic way to derive the ordered splitting based on a reference order; the algebraic method is first given in [62].

While this approach is a fully constructive method of building BCJ-compatible numerators, any crossing symmetry is broken in each of the DDM basis numerators, since the $\mathrm{OS}_{R}$ of different color ordering are not crossing symmetric. This leads to one of two computation difficulties when calculating the full integrand, either:

- each half-ladder numerator in the DDM basis must be computed individually, or

- a given half-ladder numerator must be averaged over all the reference orderings (ROaverage) to make it crossing symmetric in $\{2, \ldots, n-1\}$, which can then be freely relabeled to obtain the other half-ladder numerators.

Both approaches require an $\mathcal{O}((n-2) !)$ computation (assuming that relabeling takes negligible time comparing with computing a numerator) that must be performed after constructing a half-ladder numerator, which itself requires an $\mathcal{O}((n-1) !)$ computation, to arrive at the full DDM basis. Thus the computational complexity for the full DDM basis numerator is $\mathcal{O}[(n-1) ! \times(n-2) !]$ for the RO method.

\subsection{Recursive constructions without reference orderings for pure gluon and two-fermion numerators}

The computational complexity of the reference-ordering approach urges a more efficient construction of the kinematic factors. The fact that the crossing symmetry among $\{2, \ldots$, $n-1\}$ is recovered after RO-average hints that there should exist a new approach that solely depends on the structure of spanning trees and does not involve reference orderings at all. In this section, we present such an improved algorithm. The pure gluon and twofermion half-ladder numerators built through our new approach will be naturally crossing symmetric in the legs $\{2, \ldots, n-1\}$, removing the need for the RO-average and reducing the complexity of construction: ${ }^{8}$

$$
\mathcal{O}[(n-1) ! \times(n-2) !] \stackrel{\text { new approach }}{\longrightarrow} \mathcal{O}[(n-1) !] .
$$

\footnotetext{
${ }^{8}$ This is a very schematic comparison, and is specifically ignoring the complexity of operations that are very similar between the two procedures, e.g. processing an individual tree.
} 
The final result is equivalent to the $\mathrm{RO}$-average, but we only need to evaluate each spanning tree once. We begin by considering how to build the RO-average into simple building blocks of the increasing trees, and then present the full, recursive approach.

The first important observation is that the baseline $n \rightarrow \rho_{|\rho|} \rightarrow \cdots \rightarrow \rho_{1} \rightarrow 1$ is already independent of the reference ordering. As such we will carry its definition over to our new construction. We can then proceed to considering paths of various lengths, and eventually more complicated trees.

The easiest paths to work with are length one. They are also reference-ordering independent, so their contribution can be carried over from the traditional approach,

$$
\underset{i}{\longleftarrow}: \quad \phi_{l \rightarrow i}=\mathcal{P}(l, i)=\epsilon_{l} \cdot k_{i}
$$

for each such path. Length two paths are the first object that have reference-ordering dependence. However, the two inequivalent contributions dictated by the choice of reference orderings always enter with the same weight in the average,

$$
\underset{i}{\longleftarrow}: \phi_{l \rightarrow j \rightarrow i}=\frac{1}{2}[\mathcal{P}(l, j) \mathcal{P}(j, i)+\mathcal{P}(l, j, i)]=\frac{1}{2}\left[\left(\epsilon_{l} \cdot k_{j}\right)\left(\epsilon_{j} \cdot k_{i}\right)+\epsilon_{l} \cdot f_{j} \cdot k_{i}\right] .
$$

For this particular path factor only the relative ordering between $l$ and $j$ matters: the first term $\mathcal{P}(l, j) \mathcal{P}(j, i)$ is contributed by those reference orderings in which $j$ is before $l$ while the second term $\mathcal{P}(l, j, i)$ is from those with $l$ before $j$. Thus averaging over reference orderings assign them the same weight.

For longer paths, it becomes more computationally difficult to directly calculate the RO-average. Interestingly, there exists a recursive structure that implicitly reproduces the full RO-average. The first important insight comes from stripping off the $k_{i}$ from eqs. (3.12) and (3.13) such that each becomes a Lorentz vector $\mathcal{V}_{A}^{\mu}$

$$
\begin{aligned}
& \phi_{l \rightarrow i}=\mathcal{V}_{\{l\}} \cdot k_{i} \quad \longrightarrow \quad \mathcal{V}_{\{l\}}^{\mu}=\epsilon_{l}^{\mu} \\
& \phi_{l \rightarrow j \rightarrow i}=\mathcal{V}_{\{l \rightarrow j\}} \cdot k_{i} \quad \longrightarrow \quad \mathcal{V}_{\{l \rightarrow j\}}^{\mu}=\frac{1}{2}\left[\left(\epsilon_{l} \cdot k_{j}\right) \epsilon_{j}^{\mu}+\epsilon_{l}^{\nu} f_{j}^{\nu \mu}\right] .
\end{aligned}
$$

The subscript on $\mathcal{V}$ denotes the set of subtrees rooted on $i$. We can then make use of the renaming of eq. (3.14) in eq. (3.15) to see the first hints of the recursion

$$
\mathcal{V}_{\{l \rightarrow j\}}^{\mu}=\frac{1}{2}\left[\left(\mathcal{V}_{\{l\}} \cdot k_{j}\right) \epsilon_{j}^{\mu}+\mathcal{V}_{\{l\}}^{\nu} f_{j}^{\nu \mu}\right] .
$$

Note that we have left the linearized field strength $f_{j}^{\nu \mu}$ unexpanded in order to more easily compare with the RO-average method.

While the two-vertex case is rather trivial in the RO-average, longer chains are not. Luckily, the recursive structure continues to be simple for abitrary length chains of vertices. To see this, consider the problem of finding $\mathcal{V}_{I \rightarrow j}^{\mu}$, the partial contribution of a path $I_{1} \rightarrow$ $\cdots \rightarrow I_{n} \rightarrow j \rightarrow \cdots$ with the tensor structure "downstream" of $j$ stripped off. Inspired by eq. (3.16), we make an ansatz for the recursive vertex,

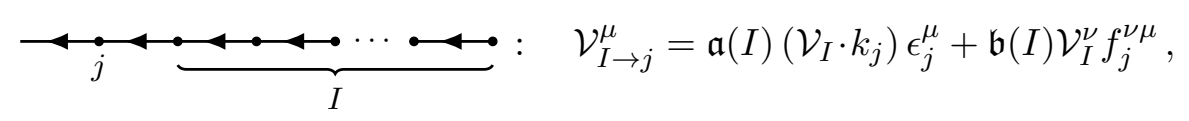




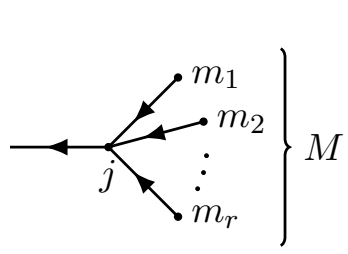

(a) Single length paths.

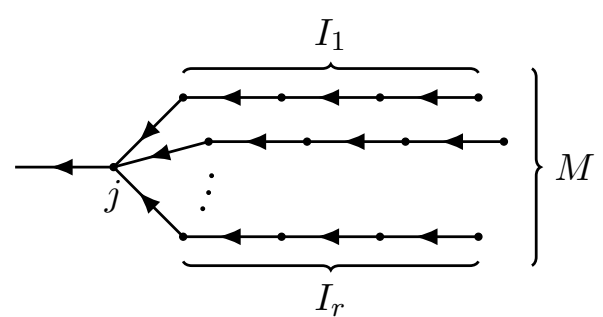

(b) Arbitrary length chains.

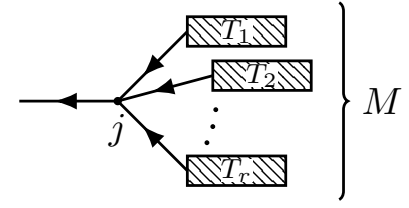

(c) Arbitrary sub-trees.

Figure 2. Merging of branches at the vertex $j$.

where $I$ is the set vertices above $j$ in the increasing tree, and $\mathfrak{a}, \mathfrak{b}$ are combinatoric coefficients that we will fix using induction. First, assume that $\mathcal{V}_{I}$ has already been properly calculated. Then, we want to incorporate the new information from $j$ in such a way that we implicitly reproduce the $\mathrm{RO}$ average along the path. In the $\mathrm{RO}$ prescription, terms of the form $\left(\mathcal{V}_{I} \cdot k_{j}\right) \epsilon_{j}^{\mu}$ will only occur for reference orderings in which $j$ precedes every element of $I$. Schematically, the ordered splitting paths are of the form

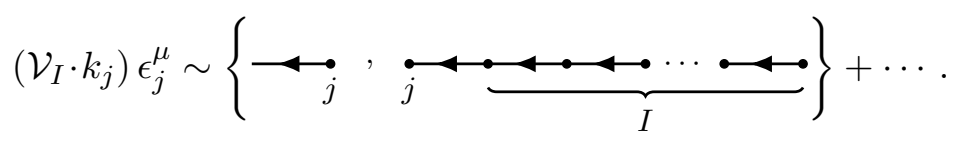

Similarly, $\mathcal{V}_{I}^{\nu} f_{j}^{\nu \mu}$ comes from the ROs in which at least one element of $I$ precedes $j$,

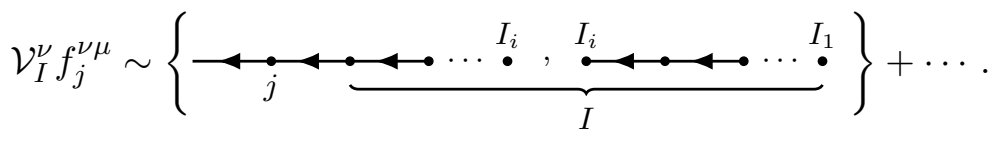

A careful counting of these permutations yields $\mathfrak{a}(I)=p|I|$ ! and $\mathfrak{b}(I)=p|I||I|$ ! with $|I|$ the number of vertices in $I$, and $p$ the joint proportionality constant. We fix this constant by demanding that, in the final result, the term involving only a product of $\epsilon \cdot k$ always carries +1 . Since this term is common to all the reference orderings, the average should not change its coefficient. As a result, $\mathfrak{a}(I)$ and $\mathfrak{b}(I)$ need to satisfy

$$
\mathfrak{a}(I)+\mathfrak{b}(I)=1 \quad \longrightarrow \quad \mathfrak{a}(I)=\frac{1}{|I|+1}, \quad \mathfrak{b}(I)=\frac{|I|}{|I|+1} .
$$

Thus, the complete expression for the $\mathcal{V}$ factor of an arbitrary length path is given by the recursive definition

$$
\mathcal{V}_{I \rightarrow j}^{\mu}=\frac{1}{|I|+1}\left[\left(\mathcal{V}_{I} \cdot k_{j}\right) \epsilon_{j}^{\mu}+|I| \mathcal{V}_{I}^{\nu} f_{j}^{\nu \mu}\right]
$$

with the termination case given in eq. (3.14). Since we have constructed these terms using inductive matching to the $\mathrm{RO}$ contributions, the final result is guaranteed to match the RO-average calculation.

The other generalization necessary to evaluate an arbitrary tree is the merger of multiple paths at a vertex, schematic examples of which are shown in figure 2 . The referenceordering approach provides an explicit splitting of the tree at such vertices, but the goal 
is to avoid such a choice. Luckily, the recursive structure can be continued to this case. The simple example is the merger of two length-one paths at a vertex. The result of RO-average is

$$
\begin{aligned}
\underbrace{l}_{j}: \phi_{\{m, l\} \rightarrow j \rightarrow i}=\mathcal{V}_{\{m, l\} \rightarrow j} \cdot k_{i}= & \frac{1}{3}[ \\
& \left(\epsilon_{m} \cdot k_{j}\right)\left(\epsilon_{l} \cdot k_{j}\right)\left(\epsilon_{j} \cdot k_{i}\right) \\
& \left.+\epsilon_{m} \cdot f_{j} \cdot k_{i}\left(\epsilon_{l} \cdot k_{j}\right)+\epsilon_{l} \cdot f_{j} \cdot k_{i}\left(\epsilon_{m} \cdot k_{j}\right)\right],
\end{aligned}
$$

in which there are three different contributions: one each from when $m$ or $l$ comes before $j$ in the reference orderings, and a third when $j$ is first among the three. Using the knowledge we have already gained from analyzing the simpler paths in eqs. (3.13) and (3.15), we can rewrite $\mathcal{V}_{\{m, l\} \rightarrow j}^{\mu}$ as

$$
\begin{aligned}
\mathcal{V}_{\{m, l\} \rightarrow j}^{\mu} & =\frac{1}{3}\left[\epsilon_{j}^{\mu} \prod_{p \in\{m, l\}} \epsilon_{p} \cdot k_{j}+\sum_{p \in\{m, l\}} \epsilon_{p}^{\nu} f_{j}^{\nu \mu} \prod_{\substack{q \in\{m, l\} \\
q \neq p}} \epsilon_{q} \cdot k_{j}\right] \\
& =\frac{1}{3}\left[\epsilon_{j}^{\mu} \prod_{p \in\{m, l\}} \mathcal{V}_{\{p\}} \cdot k_{j}+\sum_{p \in\{m, l\}} \mathcal{V}_{\{p\}}^{\nu} f_{j}^{\nu \mu} \prod_{\substack{q \in\{m, l\} \\
q \neq p}} \mathcal{V}_{\{q\}} \cdot k_{j}\right] .
\end{aligned}
$$

The use of $\prod$ and $\sum$ in eq. (3.23) makes the generalization to more incoming length-one paths straightforward: for incoming paths from vertices $M=\left\{m_{1}, \ldots, m_{r}\right\}$ we have

$$
\text { figure 2a : } \mathcal{V}_{M \rightarrow j}^{\mu}=\frac{1}{|M|+1}\left[\epsilon_{j}^{\mu} \prod_{m_{a} \in M} \mathcal{V}_{\left\{m_{a}\right\}} \cdot k_{j}+\sum_{m_{a} \in M} \mathcal{V}_{\left\{m_{a}\right\}}^{\nu} f_{j}^{\nu \mu} \prod_{\substack{m_{b} \in M \\ b \neq a}} \mathcal{V}_{\left\{m_{b}\right\}} \cdot k_{j}\right]
$$

Finally, it is necessary to generalize each $m_{a} \in M$ to an arbitrary length chain $I_{a}$, see figure 2b. Now $M$ becomes $M=\left\{I_{1}, \ldots, I_{r}\right\}$, where each $I_{a}$ is a chain as in eq. (3.21). Due to the recursive structure, most of the work was already done above in eq. (3.21): we can simply replace each $\mathcal{V}_{\left\{m_{a}\right\}}^{\mu}$ by $\mathcal{V}_{I_{a}}^{\mu}$ and adjust the relative coefficients,

$$
\text { figure 2b: } \begin{aligned}
\mathcal{V}_{M \rightarrow j}^{\mu} & =\mathfrak{a} \epsilon_{j}^{\mu} \prod_{I_{a} \in M} \mathcal{V}_{I_{a}} \cdot k_{j}+\sum_{I_{a} \in M} \mathfrak{b}_{a} \mathcal{V}_{I_{a}}^{\nu} f_{j}^{\nu \mu} \prod_{\substack{I_{b} \in M \\
b \neq a}} \mathcal{V}_{I_{b}} \cdot k_{j} \\
& =\frac{1}{1+\sum_{I_{a} \in M}\left|I_{a}\right|}\left[\epsilon_{j}^{\mu} \prod_{I_{a} \in M} \mathcal{V}_{I_{a}} \cdot k_{j}+\sum_{I_{a} \in M}\left|I_{a}\right| \mathcal{V}_{I_{a}}^{\nu} f_{j}^{\nu \mu} \prod_{\substack{I_{b} \in M \\
b \neq a}} \mathcal{V}_{I_{b}} \cdot k_{j}\right]
\end{aligned}
$$

where $\mathfrak{a}$ is the (normalized) number of reference orderings in which $j$ is before $M$, and similarly $\mathfrak{b}_{a}$ is the (normalized) number of reference orderings in which the first element in the sub-ordering of $M \cup\{j\}$ belongs to $I_{a}$. 


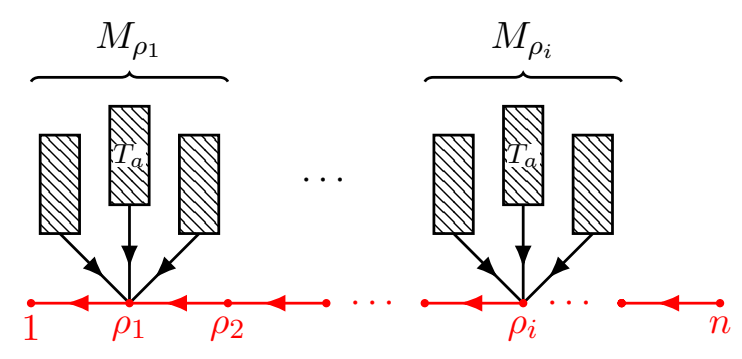

Figure 3. A typical spanning tree with the baseline shown in red, where $T_{a} \in M_{\rho_{i}}$ are the sub-trees merging at $\rho_{i}$ on the baseline.

It is important to notice that the coefficients $\mathfrak{a}$ and $\mathfrak{b}_{a}$ depend only on the number vertices in each sub-tree but not the structure. Therefore, this expression can be used to calculate any sub-trees merging at one vertex, see figure 2 c, where $M=\left\{T_{1}, \ldots, T_{r}\right\}$ and each $T_{a}$ is an arbitrary sub-tree,

$$
\text { figure 2c : } \mathcal{V}_{M \rightarrow j}^{\mu}=\frac{1}{1+\sum_{T_{a} \in M}\left|T_{a}\right|}\left[\epsilon_{j}^{\mu} \prod_{T_{a} \in M} \mathcal{V}_{T_{a}} \cdot k_{j}+\sum_{T_{a} \in M}\left|T_{a}\right| \mathcal{V}_{T_{a}}^{\nu} f_{j}^{\nu \mu} \prod_{\substack{T_{b} \in M \\ b \neq a}} \mathcal{V}_{T_{b}} \cdot k_{j}\right]
$$

due to the recursive assumption that each $\mathcal{V}_{T_{a}}$ has dressed its sub-tree correctly. Thus for the most generic sub-tree structure one only needs to correctly merge the information from each $T_{a}$ via the relative normalizations, which are not sensitive to the structure of the sub-trees.

With these new kinematic dressings, we can completely get rid of item 2 in the previous algorithm since explicit ordered splitting computations are no longer necessary. For completeness, our improved algorithm for calculating the numerator contribution $N(T)$ for a given increasing tree $T$ is:

Algorithm 2 (Recursive Evaluation). Construction of the kinematic factor for a spanning tree $T$ recursively.

1. For the path $n \rightarrow \rho_{|\rho|} \rightarrow \ldots \rho_{1} \rightarrow 1$, assign a baseline factor $W(1, \rho, n)$. Note that $n \rightarrow 1$ and thus $\rho=\emptyset$ is allowed, which contributes $W(1, n)$.

2. For each vertex $\left\{1, \rho_{1}, \ldots, \rho_{|\rho|}\right\}$ on the baseline, identify $M_{\rho_{i}}$ as the set of sub-trees merging at $\rho_{i}$, see figure 3. For notational convenience we define $\rho_{0}=1$.

3. Calculate $\mathcal{V}$ from each sub-tree $T_{a} \in M_{\rho_{i}}$ using eq. (3.26) and assemble the result:

$$
N(T)=W(1, \rho, n) \prod_{i=0}^{|\rho|} \prod_{T_{a} \in M_{\rho_{i}}} \mathcal{V}_{T_{a}} \cdot k_{\rho_{i}} .
$$

This algorithm only needs to process a single spanning tree $T$ once, and directly generates the crossing symmetric numerator in $\{2, \ldots, n-1\}$, thus eliminating the $\mathcal{O}[(n-2) !]$ 
workload due to the RO-average, as advertised at the beginning of this subsection. We have explicitly checked agreement with numerators generated by RO-average through seven points, and numerically checked that they reproduce correct amplitudes through nine points. ${ }^{9}$ We note that in our numerators $k_{n}$ does not appear by construction, and neither does $\epsilon_{n} \cdot k_{1}$. Thus our numerators are naturally in a basis of momentum conservation.

While the procedure was designed with pure Yang-Mills trees in mind, we can recall from eqs. (2.5) and (2.7) that all terms that care about 1 and $n$ being fermions are localized to the baseline function $W_{2 \mathrm{f}}$. The rest of the kinematic structure is exactly identical to the pure Yang-Mills case. Thus, this algorithm also constructs the half-ladder numerators with two fermions.

\subsection{Four-point YM example}

With the general principle under control, we'll now turn to an explicit example, and demonstrate some of the functionality of the MAтHEMATicapackage. We'll construct the DDM basis numerator $N(1,2,3,4)$ using our new method, and then show that it indeed gives the correct numerator for $N(1,3,2,4)$ via relabeling.

The first step for building the numerators is to enumerate $\operatorname{IT}(1,2,3,4)$, which is implemented by the function increasingTrees:

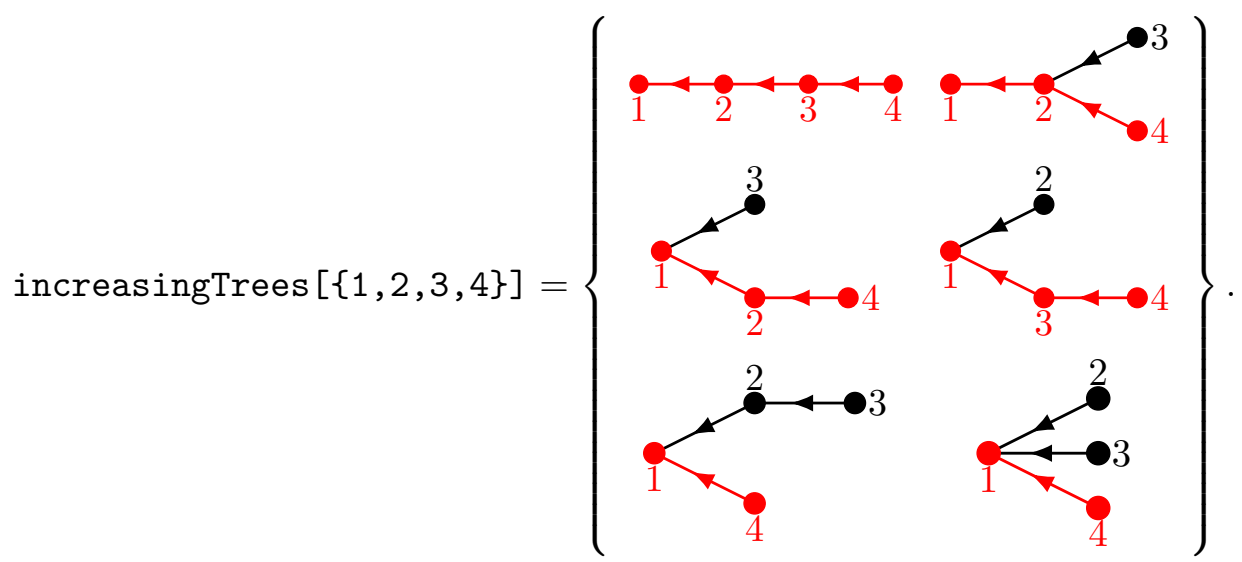

Then we need to dress each of the trees with their appropriate kinematic factors. This is handled by the function itNumer. For the simplest example, we have

$$
\text { itNumer }[1+2
$$

The most complicated case is actually entirely covered by eq. (3.16):

$$
\text { itNumer }\left[1<_{0}^{2} \cdot 3,4\right]=\frac{1}{2}\left(\left(\epsilon_{3} \cdot k_{2}\right)\left(\epsilon_{2} \cdot k_{1}\right)+\epsilon_{3} \cdot f_{2} \cdot k_{1}\right) W_{\text {gluon }}(1,4)
$$

\footnotetext{
${ }^{9}$ The new technique can compute a nine-point half-ladder numerator in a few minutes, while the old algorithm given in section 3.1 struggles at seven points. Our new approach is used extensively for construction and checks in a parallel study of one-loop integrands from forward limits [78].
} 
Combining all of the contributions together gives us

$$
\begin{aligned}
N(1,2,3,4)= & W_{\text {gluon }}(1,2,3,4)+W_{\text {gluon }}(1,2,4)\left(\epsilon_{3} \cdot k_{2}+\epsilon_{3} \cdot k_{1}\right)+W_{\text {gluon }}(1,3,4) \epsilon_{2} \cdot k_{1} \\
& +W_{\text {gluon }}(1,4)\left(\frac{1}{2}\left(\left(\epsilon_{3} \cdot k_{2}\right)\left(\epsilon_{2} \cdot k_{1}\right)+\epsilon_{3} \cdot f_{2} \cdot k_{1}\right)+\left(\epsilon_{2} \cdot k_{1}\right)\left(\epsilon_{3} \cdot k_{1}\right)\right)
\end{aligned}
$$

We can do a similar calculation to find the $(1,3,2,4)$ ordering

$$
\begin{aligned}
N(1,3,2,4)= & W_{\text {gluon }}(1,3,2,4)+W_{\text {gluon }}(1,3,4)\left(\epsilon_{2} \cdot k_{3}+\epsilon_{2} \cdot k_{1}\right)+W_{\text {gluon }}(1,2,4) \epsilon_{3} \cdot k_{1} \\
& +W_{\text {gluon }}(1,4)\left(\frac{1}{2}\left(\left(\epsilon_{2} \cdot k_{3}\right)\left(\epsilon_{3} \cdot k_{1}\right)+\epsilon_{2} \cdot f_{3} \cdot k_{1}\right)+\left(\epsilon_{3} \cdot k_{1}\right)\left(\epsilon_{2} \cdot k_{1}\right)\right)
\end{aligned}
$$

which shows that, as claimed, the two numerators are just relabelings of each other under $(2 \leftrightarrow 3)$. The function ptTreeNumer will fully calculate an n-point tree numerator, and relabel and dress the numerator with the appropriate Parke-Taylor factor

$$
\text { ptTreeNumer }[4]=N(1,2,3,4) \mathrm{PT}(1,2,3,4)+N(1,3,2,4) \mathrm{PT}(1,3,2,4) .
$$

As discussed in section 2.1, this also builds the correct numerator for two fermions, $N_{2 \mathrm{f}}$, by replacing $W_{\text {gluon }} \rightarrow W_{2 \mathrm{f}}$.

\subsection{Recursive constructions for multi-trace}

The recursive construction we described in section 3.2 and algorithm 2 can be extended to also handle multi-trace YMS numerators. We will work out how to build numerators of the form $N_{\mathrm{MT}}(1, \beta, n)$, with an additional set of specified scalar traces $\tau_{i}$. First using the baseline expansion discussed in section 2.2, we can put the legs 1 and $n$ to the endpoints of a single scalar trace, see eqs. (2.16) and (2.30). Then we can work out the numerators following the process first proposed in ref. [66], in particular, a spanning-tree algorithm given in section 10.3 (see also appendix A of ref. [34]). However, this algorithm is RObased and thus crossing symmetry is not manifest. In this section, we develop additional refinements that restore crossing symmetry to the numerators, in line with what we did for the pure Yang-Mills numerators in section 3.2. Similar to the pure Yang-Mills case, the process involves two steps, constructing spanning trees and dressing with kinematic factors. As discussed in section 2.2, the gluons and scalar traces are generally treated on the same footing.

First, we discuss the structure of the increasing trees to dress, and more generally the interplay between trace orderings and numerator ordering. The analog of the increasingtree ordering used in eq. (3.6) is obtained by applying the coarse-grained $\mathscr{O}$ function $(2.22)$ to the entire numerator ordering $(1, \beta, n)$,

$$
\mathscr{O}_{(1, \beta, n)}^{\mathrm{cg}}=\left(\lambda_{1}, \lambda_{2}, \ldots, \lambda_{\mathrm{N}}\right) \in S_{\left\{\mathrm{t}_{1}, \mathrm{t}_{2}, \ldots, \mathrm{t}_{\mathrm{N}}\right\}},
$$

where each $\lambda_{i}$ represents a scalar trace or a gluon. It is an ordering of our particle inventory $\left\{\mathrm{t}_{1}, \mathrm{t}_{2}, \ldots, \mathrm{t}_{\mathrm{N}}\right\}=\left\{\tau_{1}, \tau_{2}, \ldots, \tau_{m}\right\} \cup G$ with $\mathrm{N}=m+|G|$, where scalar traces $\tau_{i}$ are treated on the same footing as a gluon in $G$. In addition, the first entry $\lambda_{1}$ must be the trace that 
leg 1 belongs to, or the leg 1 itself if it is a gluon. We can then construct all the increasing trees according to eq. (3.6) and write

$$
N_{\mathrm{MT}}(1, \beta, n)=\sum_{T \in \mathrm{IT}\left(\mathscr{O}_{(1, \beta, n)}^{\mathrm{cg}}\right)} N_{\mathrm{MT}}(T) .
$$

The vertices of each tree $T$ are $t_{i}$ 's, which can either be a scalar trace or a gluon.

Now that the increasing trees are laid out, we can begin assigning their kinematic dressings. To evaluate $N_{\mathrm{MT}}(T)$, the first step is to extract the baseline from a tree $T$. Suppose $1 \in \mathrm{t}_{1}$ and $n \in \mathrm{t}_{m}$, we first extract the path $\left(\mathrm{t}_{1}, \lambda_{1}, \ldots, \lambda_{|B|}, \mathrm{t}_{m}\right)$ that connects them in $T$. If $n$ is a gluon, then $t_{m}=n$ must be a leaf, while this is not necessary when $t_{m}$ is a scalar trace. Moreover, $\mathrm{t}_{m}$ can coincide with $\mathrm{t}_{1}$. This happens when 1 and $n$ belong to the same scalar trace to begin with. For this case, the path consists of just a single vertex $t_{1}$. The baseline ordering $(1, \rho, n)$ is then obtained by restricting the numerator color ordering $(1, \beta, n)$ to $\left(\mathrm{t}_{1}, \lambda_{1}, \ldots, \lambda_{|B|}, \mathrm{t}_{m}\right)$,

$$
(1, \rho, n)=\left.(1, \beta, n)\right|_{\left(\mathrm{t}_{1}, \lambda_{1}, \ldots, \lambda_{|B|}, \mathrm{t}_{m}\right)},
$$

which is exactly the baseline discussed in section 2.2. We can thus dress it with the kinematic factor $W_{\mathrm{MT}}(1, \rho, n)$ defined in eq. (2.27). On the other hand, given a baseline $(1, \rho, n)$, we can recover the path $\left(\mathrm{t}_{1}, \lambda_{1}, \ldots, \lambda_{|B|}, \mathrm{t}_{m}\right)$, which we call the coarse-grained baseline, by using the coarse-grained $\mathscr{O}$ function,

$$
\left(\mathrm{t}_{1}, \lambda_{1}, \ldots, \lambda_{|B|}, \mathrm{t}_{m}\right)=\mathscr{O}_{(1, \rho, n)}^{\mathrm{cg}} .
$$

Eq. (3.51) in the following section provides explicit examples of this baseline identification. Now similar to eq. (3.27), we can write $N_{\mathrm{MT}}(T)$ as

$$
N_{\mathrm{MT}}(T)=W_{\mathrm{MT}}(1, \rho, n) \prod_{\mathbf{i} \in \mathscr{O}_{(1, \rho, n)}^{\mathrm{cg}}} \prod_{T_{a} \in M_{\mathrm{i}}} \mathcal{V}_{T_{a}} \cdot \mathcal{K}_{\mathbf{i}, T_{a}}
$$

where $M_{\mathrm{i}}$ is the set of sub-trees merging at the vertex $\mathrm{i}$ on the coarse-grained baseline $\mathscr{O}_{(1, \rho, n)}^{\mathrm{cg}}$. The kinematic factor associated to each sub-tree $T_{a} \in M_{\mathrm{i}}$ is $\mathcal{V}_{T_{a}} \cdot \mathcal{K}_{\mathrm{i}, T_{a}}$, where $\mathcal{K}$ will be defined below in eq. (3.43). The vertex function $\mathcal{V}$ can be evaluated by RObased methods [34, 66], but in the following, we will propose an improved algorithm that automatically recovers the crossing symmetry.

We denote $M=\left\{T_{1}, T_{2}, \ldots, T_{r}\right\}$ as the set of sub-trees merging at the vertex $\mathrm{t}_{j}$. By construction the vertices of our spanning trees are labeled by $\left\{\mathrm{t}_{1}, \ldots, \mathrm{t}_{\mathrm{N}}\right\}$. The recursive structure of $\mathcal{V}$ when including the vertex $\mathrm{t}_{j}$ takes a form very similar to eq. (3.26),

$$
\mathcal{V}_{M \rightarrow \mathrm{t}_{j}}^{\mu}=\frac{1}{1+\sum_{T_{a} \in M}\left|T_{a}\right|}\left[b_{\mathrm{t}_{j}}^{\mu} \prod_{T_{a} \in M} \mathcal{V}_{T_{a}} \cdot \mathcal{K}_{\mathrm{t}_{j}, T_{a}}+\sum_{T_{a} \in M}\left|T_{a}\right| \mathcal{V}_{T_{a}}^{\nu} \mathcal{T}_{\mathrm{t}_{j}, T_{a}}^{\nu \mu} \prod_{\substack{T_{b} \in M \\ b \neq a}} \mathcal{V}_{T_{b}} \cdot \mathcal{K}_{\mathrm{t}_{j}, T_{a}}\right],
$$


where $\left|T_{a}\right|$ counts the number of vertices in $T_{a}$. The combinatoric coefficients here are the same as eq. (3.26) for pure gluon cases since scalar traces are treated as a single object on the same footing as gluons. The path-begin factor $b_{\mathbf{t}_{j}}^{\mu}$ is given by

$$
b_{\mathrm{t}_{j}}^{\mu}= \begin{cases}\frac{1}{\left|\mathrm{t}_{j}\right|} \mathfrak{s g n}_{a_{j}, \omega_{j}, b_{j}}^{\mathrm{t}_{j}}\left(-k_{a_{j}}^{\mu}\right) & \mathrm{t}_{j} \text { a trace } \\ \epsilon_{\mathrm{t}_{j}}^{\mu} & \mathrm{t}_{j} \text { is a gluon }\end{cases}
$$

where $\left(a_{j}, \omega_{j}, b_{j}\right)$ the sub-ordering of the elements in $\mathrm{t}_{j}$ in the numerator ordering $(1, \beta, n)$. The effect of RO-average is encoded in the prefactor $\frac{1}{\left|t_{j}\right|}$. This prescription recovers the crossing symmetry within the trace $t_{j}$. We note that in the RO approach given in $[34,66]$, one has to pick the same $a_{j}$ across when $\mathrm{t}_{j}$ appears in the path-begin factor for all the numerator ordering, which breaks the manifest cyclicity of the scalar traces. Next, the through factor $\mathcal{T}_{\mathrm{t}_{j}, T_{a}}^{\mu \nu}$. At this point, it is useful to generalize $\mathcal{B}$ to take trees (multiple traces) after the vertical bar via

$$
\mathcal{B}_{(1, \beta, n)}\left(\mathrm{t}_{i} \mid T_{a}\right) \equiv \bigcap_{\mathrm{t}_{l} \in T_{a}} \mathcal{B}_{(1, \beta, n)}\left(\mathrm{t}_{j} \mid \mathrm{t}_{l}\right)
$$

that is, $\mathcal{B}_{(1, \beta, n)}\left(\mathrm{t}_{i} \mid T_{a}\right)$ consists of the elements in $\mathrm{t}_{i}$ that come before everything in $T_{a}$ in the numerator ordering $(1, \beta, n)$. Then $\mathcal{T}_{\mathfrak{t}_{j}, T_{a}}$ is given by

$$
\mathcal{T}_{\mathrm{t}_{j}, T_{a}}^{\mu \nu}= \begin{cases}\mathfrak{s g n}_{a_{j}, \omega_{j}, b_{j}}^{\mathrm{t}_{j}}\left(-k_{b_{j}}^{\mu} k_{a_{j}}^{\nu}\right) & \mathrm{t}_{j} \text { is a trace and } \mathcal{B}_{(1, \beta, n)}\left(\mathrm{t}_{j} \mid T_{a}\right)=\mathrm{t}_{j} \\ 0 & \mathrm{t}_{j} \text { is a trace and } \mathcal{B}_{(1, \beta, n)}\left(\mathrm{t}_{j} \mid T_{a}\right) \subsetneq \mathrm{t}_{j} . \\ f_{\mathrm{t}_{j}}^{\mu \nu} & \mathrm{t}_{j} \text { is a gluon }\end{cases}
$$

Note that it takes a similar form to the $\mathcal{T}$ factor used in the baseline factor $W_{\mathrm{MT}}(1, \rho, n)$, see eq. (2.28a). The $\mathfrak{s g n}$ function appearing in eqs. (3.40) and (3.42) ensures that $N_{\mathrm{MT}}(1, \beta, n)$ is nonzero if and only if the numerator ordering $(1, \beta, n)$ is KK-compatible to every scalar trace. Finally, the path-end factor $\mathcal{K}_{\mathrm{t}_{j}, T_{a}}^{\mu}$ is given by

$$
\mathcal{K}_{\mathrm{t}_{j}, T_{a}}^{\mu}=\sum_{m \in \mathcal{B}_{(1, \beta, n)}\left(\mathrm{t}_{j} \mid T_{a}\right)} k_{m}^{\mu},
$$

the total momentum from $\mathrm{t}_{j}$ that comes before everything in $T_{a}$. The factor $\mathcal{K}$ is actually the same as the one used in the RO approach [34,66]. Finally, we note that the proof of eq. (3.39) resembles that of section 3.2, which we will not repeat here.

\subsection{Multi-trace example}

We'll now walk through a few examples of putting the multi-trace expansion into practice. First, we'll calculate $N(1,2,3,4,5,6,7,8)$ where the traces are decomposed and colorordered as

$$
\tau_{1}=(1,8) \quad \tau_{2}=(2,3) \quad \tau_{3}=(4,6) \quad \tau_{4}=(5,7) .
$$

Since 1 and 8 are already in the same trace, we don't need to use the full power of the baseline recursion in this case, and can instead focus on the kinematic factors immediately. 
The increasing trees that contribute to this numerator will have $\tau_{1}$ as the baseline, and the remaining $\tau$ s as vertices along the trees. In this particle ordering, the traces are shuffled via

$$
(1, \beta, 8)=(1,2,3, \underbrace{2,}_{\tau_{2}}, \underbrace{\frac{\tau_{3}}{5,6}}_{\tau_{4}}, 7,8) .
$$

so the increasing trees are ordered via $\tau_{2}$ before $\tau_{3}$ before $\tau_{4}$. The six increasing trees that build this numerator can be generated using

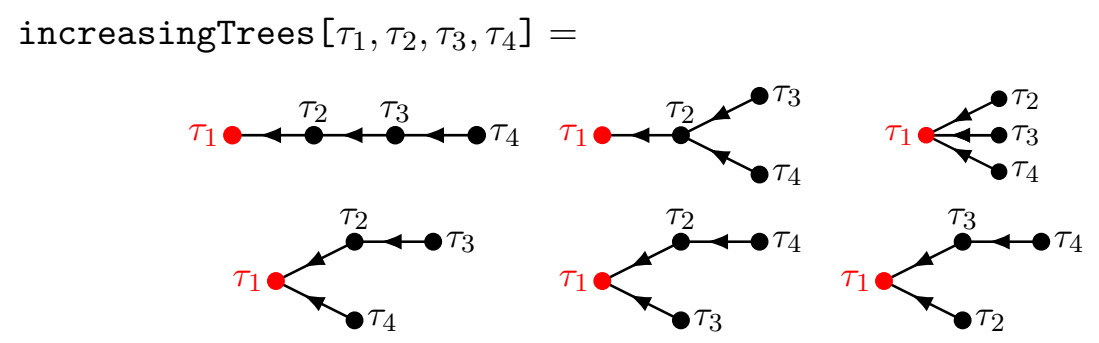

Since $\mathcal{B}\left(\tau_{3} \mid \tau_{4}\right)=(4) \neq \tau_{3}$, the two trees with edge $\tau_{4} \rightarrow \tau_{3}$ are in the $\mathcal{T}^{\mu \nu}=0$ case of eq. (3.42). Other than that important caveat, the last five of these trees can be evaluated by directly adapting various examples we covered in the YM construction. The first tree will be a good example application of the recursive structure. Using the kinematic dressings from section 3.4 in the recursion, we have

$$
\begin{aligned}
& N\left(\tau_{1}\right. \\
& \quad=-W_{\mathrm{MT}}\left(\tau_{1}\right) \frac{1}{3} k_{1}^{\mu}\left(\frac{1}{2} k_{2}^{\mu}\left(k_{23} \cdot \mathcal{V}_{\tau_{3} \tau_{4}}\right)+2 k_{2}^{\mu}\left(k_{3} \cdot \mathcal{V}_{\tau_{3} \tau_{4}}\right)\right) \\
& \quad=W_{\mathrm{MT}}\left(\tau_{1}\right)\left(k_{1} \cdot k_{2}\right) \frac{1}{6}\left[\left(k_{23}+4 k_{3}\right)^{\nu} \frac{1}{2}\left(\frac{1}{2} k_{4}^{\nu}\left(k_{4} \cdot \mathcal{V}_{\tau_{4}}\right)+k_{4}^{\nu}(0)\right)\right] \\
& \quad=-\frac{1}{48}\left(k_{1} \cdot k_{2}\right)\left(k_{4} \cdot k_{5}\right)\left(k_{23} \cdot k_{4}+4 k_{3} \cdot k_{4}\right),
\end{aligned}
$$

where $W_{\mathrm{MT}}\left(\tau_{1}\right)=1$. We do not directly provide a function to calculate individual tree dressings for YMS due to the need to calculate $\mathcal{B}$ and $\mathscr{O}$ from the full numerator ordering. However, the full result from combining all of the increasing trees can be calculated via the package using

$$
\begin{aligned}
& \text { multiTraceNumer }[\{1,2,3,4,5,6,7,8\},\{\{1,8\},\{2,3\},\{4,6\},\{5,7\}\}]= \\
& -\frac{1}{48}\left(k_{1} \cdot k_{2}\right)\left[2\left(k_{2} \cdot k_{4}\right)\left(k_{2} \cdot k_{5}\right)+6\left(k_{2} \cdot k_{5}\right)\left(k_{3} \cdot k_{4}\right)+3\left(k_{1} \cdot k_{5}\right)\left(k_{2} \cdot k_{4}+3 k_{3} \cdot k_{4}\right)\right. \\
& +6\left(k_{2} \cdot k_{4}\right)\left(k_{3} \cdot k_{5}\right)+10\left(k_{3} \cdot k_{4}\right)\left(k_{3} \cdot k_{5}\right)+\left(k_{2} \cdot k_{4}\right)\left(k_{4} \cdot k_{5}\right)+5\left(k_{3} \cdot k_{4}\right)\left(k_{4} \cdot k_{5}\right) \\
& \left.+3\left(k_{1} \cdot k_{4}\right)\left(2\left(k_{1} \cdot k_{5}\right)+\left(k_{2} \cdot k_{5}\right)+3\left(k_{3} \cdot k_{5}\right)+\left(k_{4} \cdot k_{5}\right)\right)\right] \text {. }
\end{aligned}
$$

Next, we look at a case involving gluons that demonstrates the baseline expansion. The goal is to calculate $N(1,2,3,4,5,6)$ with

$$
\tau_{1}=(1,2) \quad \tau_{2}=(3,4) \quad G=\{5,6\} .
$$


Since we don't have a scalar trace of the form $(1 \ldots n)$, we need to perform the sum over pushing $\tau_{2}$ and 5 into the baseline with $\tau_{1}$ and 6 . The various expansions of the baseline are given by

$$
\begin{aligned}
W_{\mathrm{MT}}\left(\tau_{1}, 6\right) & =k_{2} \cdot \epsilon_{6} & W_{\mathrm{MT}}\left(\tau_{1}, 5,6\right) & =-k_{2} \cdot f_{5} \cdot \epsilon_{6} \\
W_{\mathrm{MT}}\left(\tau_{1}, \tau_{2}, 6\right) & =-\left(k_{2} \cdot k_{3}\right)\left(k_{4} \cdot \epsilon_{6}\right) & W_{\mathrm{MT}}\left(\tau_{1}, \tau_{2}, 5,6\right) & =\left(k_{2} \cdot k_{3}\right)\left(k_{4} \cdot f_{5} \cdot \epsilon_{6}\right)
\end{aligned}
$$

which correspond to the increasing trees

$$
\begin{aligned}
& W_{\mathrm{MT}}\left(\tau_{1}, 6\right): \tau_{1} \stackrel{\tau_{2}}{\overbrace{\bullet}} \cdot 5 \\
& \tau_{1} \overbrace{6}^{\tau_{2}} \\
& W_{\mathrm{MT}}\left(\tau_{1}, 5,6\right): \tau_{1} \stackrel{\tau_{2}}{\tau_{5}} \\
& W_{\mathrm{MT}}\left(\tau_{1}, \tau_{2}, 6\right): \tau_{1}
\end{aligned}
$$

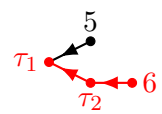

We can get the combined result by again using multiTraceNumer, dropping the gluons from the trace specification

$$
\begin{aligned}
& \text { multi TraceNumer }[\{1,2,3,4,5,6\},\{\{1,2\},\{3,4\}\}]= \\
& \qquad \frac{1}{2} W_{\mathrm{MT}}\left(\tau_{1}, 6\right)\left(2 \epsilon_{5} \cdot k_{1}+2 \epsilon_{5} \cdot k_{2}+\epsilon_{5} \cdot k_{3}+3 \epsilon_{5} \cdot k_{4}\right)\left(k_{1} \cdot k_{3}+k_{2} \cdot k_{3}\right) \\
& \quad-W_{\mathrm{MT}}\left(\tau_{1}, \tau_{2}, 6\right)\left(\epsilon_{5} \cdot k_{1234}\right)-\frac{1}{2} W_{\mathrm{MT}}\left(\tau_{1}, 5,6\right)\left(k_{1} \cdot k_{3}+k_{2} \cdot k_{3}\right)+W_{\mathrm{MT}}\left(\tau_{1}, \tau_{2}, 5,6\right) .
\end{aligned}
$$

\section{Introducing masses}

Since the numerator construction we have described is dimensionally agnostic (up to the choice of fermion wavefunctions), it is straightforward to obtain numerators of minimallycoupled massive theories through a dimensional compactification. Such a construction is equivalent to a spontaneous symmetry breaking [83]. In this section, we will work with the mostly minus metric.

In particular, it is very convenient to introduce a mass to particles 1 and $n$ that sit at the end points of the baseline. To construct the desired massive numerators in $4 \mathrm{D}$, we start by picking special kinematics in $6 \mathrm{D}$,

$$
K_{1}=\left(\begin{array}{c}
k_{1} \\
0 \\
-m
\end{array}\right), \quad K_{2}=\left(\begin{array}{c}
k_{2} \\
0 \\
0
\end{array}\right), \quad \ldots \quad K_{n}=\left(\begin{array}{c}
k_{n} \\
0 \\
m
\end{array}\right) .
$$

where $K_{i}$ are $6 \mathrm{D}$ vectors, and $k_{i}$ are $4 \mathrm{D}$ vectors. The $6 \mathrm{D}$ massless conditions $K_{1}^{2}=K_{n}^{2}=0$ impose the $4 \mathrm{D}$ massive conditions $k_{1}^{2}=k_{n}^{2}=m^{2}$. For scalars, no modifications are needed as their external wavefunctions are trivial. The gluon polarization vectors are all kept in $4 \mathrm{D}, \mathscr{E}_{i}=\left(\epsilon_{i}, 0,0\right)$, and transverse to the momentum, $K_{i} \cdot \mathscr{E}_{i}=k_{i} \cdot \epsilon_{i}=0$. 
To build up the explicit embedding of massive 4D spinors into massless $6 \mathrm{D}$ spinors, one can follow the technique of $[4,84]$,

$$
\begin{aligned}
& \left|1_{a}\right\rangle^{A}=\left(\begin{array}{cc}
\left|1^{+}\right\rangle & \left|1^{-}\right\rangle \\
\left.\mid 1^{+}\right] & \left.\mid 1^{-}\right]
\end{array}\right)=\left(u^{+}\left(k_{1}\right), u^{-}\left(k_{1}\right)\right), \\
& \left.\mid 1_{\dot{a}}\right]_{A}=\left(\begin{array}{l}
-\left\langle 1^{+}\right|\left\langle 1^{-}\right| \\
-\left[1^{+} \mid\left[1^{-} \mid\right.\right.
\end{array}\right)=\left(-\bar{v}^{+}\left(k_{1}\right), \bar{v}^{-}\left(k_{1}\right)\right), \\
& \left|n_{a}\right\rangle^{A}=\left(\begin{array}{cc}
-\left|n^{+}\right\rangle & \left|n^{-}\right\rangle \\
\left.\mid n^{+}\right] & \left.-\mid n^{-}\right]
\end{array}\right)=\left(v^{+}\left(k_{n}\right),-v^{-}\left(k_{n}\right)\right), \\
& \left.\mid n_{\dot{a}}\right]_{A}=\left(\begin{array}{cc}
\left\langle n^{+}\right| & \left\langle n^{-}\right| \\
-\left[n^{+} \mid\right. & -\left[n^{-} \mid\right.
\end{array}\right)=\left(-\bar{u}^{+}\left(k_{n}\right), \bar{u}^{-}\left(k_{n}\right)\right),
\end{aligned}
$$

where $\left|i^{ \pm}\right\rangle$and $\mid i^{ \pm}$] are massive spinor helicity variables [85], and $u$ and $v$ are usual momentum-space Dirac spinors. The explicit representation of $6 \mathrm{D}$ spinors depends on the choice of $6 \mathrm{D}$ gamma matrices, and here we use the one given in the appendix $\mathrm{A}$ of ref. [80]. ${ }^{10}$ Our definition of Dirac spinors in terms of massive spinor helicity variables follows ref. [86], where one can also find explicit formulas for these quantities.

We note that all the formal expressions for our numerators are unchanged under the uplift (4.1), except that one needs to use massive kinematics when evaluating. In fact, the only way to introduce an explicit mass term is via $K_{1} \cdot K_{n}=k_{1} \cdot k_{n}+m^{2}$ while $K_{i} \cdot K_{j}=k_{i} \cdot k_{j}$ for all the rest. Our numerators are constructed to only contain the wavefunction of particle $n$ but not the momentum, such that the product $K_{1} \cdot K_{n}$ will not naturally appear unless one deliberately swaps it in via momentum conservation.

\section{Conclusion and outlook}

In this paper we presented a review of the current state-of-the-art for calculating YM tree amplitudes in the CHY formalism. We identified a computational complexity that results from the requirement of the previous methods, summarized in algorithm 1 , to define an ordered splitting of paths in order to build kinematic factors, which in turn builds numerators that are not crossing symmetric. To overcome this complexity, we developed a new method of assigning kinematic factors to increasing trees that incorporates all contributions from different ordered splittings implicitly, rather than via an explicit afterthe-fact average. This new method, summarized in algorithm 2, is built around a recursive processing of the underlying spanning trees, allowing the required kinematic factors to be determined at each vertex by using a small amount of information from the previous steps in the recursion. One of the benefits of the new approach is the restoration of crossing symmetry at every step in the calculation, without needing to process the same tree multiple times. Our new numerator construction is now only a function of external particle ordering, and has no dependence on reference orderings. The new method was also straightforward to generalize to include full multi-trace YMS numerators or two-fermion numerators. Since

\footnotetext{
${ }^{10}$ This is our motivation for putting the mass into the sixth slot instead of the fifth in eq. (4.1), as this choice will lead to a simpler embedding of fermion wavefunctions.
} 
the numerators we construct are in the DDM basis, they can be double-copied immediately to yield the corresponding numerators for Einstein-Yang-Mills.

A related benefit of the new method is a signficant improvement in the accesible multiplicity of tree amplitudes. While the RO based methods require processing all of the spanning trees again for each numerator in eqs. (1.2) and (1.3), the new algorithm can simply relabel a prototype numerator. As such our new method is efficient enough that we can easily calculate 8 point YM trees, where two of the external legs can be swapped out for fermions or scalars instead; we have been able to calculate individual crossing symmetric tree numerators up to 10 points without significant difficulty. For 7 and fewer external particles, we have explicitly compared our results with the RO average. At 8 points and beyond, we have verified our results by comparison to a direct numerical calculation of eq. (2.1) on certain solutions of the scattering equations. This ability to calculate highpoint trees with super-partners has been incredibly useful in the study of forward limits, which we leave to its own paper [78].

The fermionic baseline functions are the only piece of the calculation that depend on the dimension of interest. Thus, we described one method of using dimensional compactification to introduce a masses to the 2 singled out particles labeled 1 and $n$. We hope that the combination of double-copy compatibility, potentially massive particles, and calculation efficiency will be of interest to the growing application of amplitudes techniques to classical gravity, for instance as input into the unitarity cuts approach of constructing relevant loop amplitudes [13, 15, 16, 19-21].

Finally, we provide a MATHEMATICApackage the implements the recursive numerator construction. Appendix B and the examples in sections 3.3 and 3.5 have more details about the package and its usage.

\section{Acknowledgments}

We would like to thank Song He and Oliver Schlotterer for sharing unpublished notes related to the fermionic expansion eqs. (2.7), (2.8a) and (2.8b). We would additionally like to thank Song He, Henrik Johansson, and Oliver Schlotterer for comments on the manuscript, and Maor Ben-Shahar and Gregor Kälin for testing the MAthematicapackage. AE is supported by the Knut and Alice Wallenberg Foundation under KAW 2018.0116, From Scattering Amplitudes to Gravitational Waves. FT is supported in part by the Knut and Alice Wallenberg Foundation under grant KAW 2013.0235, and the Ragnar Söderberg Foundation (Swedish Foundations' Starting Grant). Bibliography produced in part with the help of fillTEX [87].

\section{A CHY integrands and scattering amplitudes}

In this appendix, we provide the explicit forms of the CHY integrands used in the main text and the normalizations used to match Feynman diagram results. Throughout the paper, 
we use the following definition for the Pfaffian of a $(2 n) \times(2 n)$ antisymmetric matrix $X$,

$$
\operatorname{Pf}(X) \equiv(-1)^{\frac{n(n+1)}{2}} \frac{1}{2^{n} n !} \sum_{\sigma \in S_{2 n}} \operatorname{sign}(\sigma) \prod_{i=1}^{n} X_{\sigma_{2 i-1} \sigma_{2 i}} .
$$

The sign factor $(-1)^{\frac{n(n+1)}{2}}$ is not present in the usual definition of a Pfaffian [88], but convenient for our purposes.

In the pure gluon integrand (2.4), the reduced Pfaffian is defined as

$$
\operatorname{Pf}^{\prime}(\Psi) \equiv \frac{(-1)^{i+j+n-1}}{\sigma_{i j}} \operatorname{Pf}\left(\Psi_{i j}^{i j}\right), \quad 1 \leqslant i<j \leqslant n .
$$

where the $2 n \times 2 n$ antisymmetric matrix $\Psi$ is given in the block form,

$$
\Psi=\left(\begin{array}{cc}
\mathrm{A} & -\mathrm{C}^{T} \\
\mathrm{C} & \mathrm{B}
\end{array}\right) .
$$

The three $n \times n$ blocks $\mathrm{A}, \mathrm{B}$ and $\mathrm{C}$ are given by

$$
\begin{array}{lll}
\mathrm{A}_{i j}=\frac{k_{i} \cdot k_{j}}{\sigma_{i j}}, & \mathrm{~B}_{i j}=\frac{\epsilon_{i} \cdot \epsilon_{j}}{\sigma_{i j}}, & \mathrm{C}_{i j}=\frac{\epsilon_{i} \cdot k_{j}}{\sigma_{i j}}, \\
\mathrm{~A}_{i i}=0, & \mathrm{~B}_{i i}=0, & \mathrm{C}_{i i}=-\sum_{\substack{j=1 \\
j \neq i}}^{n} \mathrm{C}_{i j} .
\end{array}
$$

The polarization vectors are normalized as $\epsilon_{i} \cdot \epsilon_{i}^{*}=1$. The matrix $\Psi_{i j}^{i j}$ is obtained from $\Psi$ by deleting the $i$-th and $j$-th row and column. On the support of the scattering equations, $\mathrm{Pf}^{\prime}(\Psi)$ is independent of the choice of $i$ and $j$. The $\Psi_{G}$ that appears in the baseline expansion (2.5) and the single-trace integrand (2.12) is a $2|G| \times 2|G|$ antisymmetric submatrix of $\Psi$, obtained by restricting the rows and columns in the A, B and C blocks to the set $G$,

$$
\Psi_{G}=\left(\begin{array}{cc}
\mathrm{A}_{G} & -\left(\mathrm{C}_{G}\right)^{T} \\
\mathrm{C}_{G} & \mathrm{~B}_{G}
\end{array}\right)
$$

where $\left(\mathrm{X}_{G}\right)_{i j}=\mathrm{X}_{i j}$ for $\mathrm{X}=\mathrm{A}, \mathrm{B}, \mathrm{C}$ and $i, j \in G$.

The multi-trace integrand $\mathcal{I}_{n}\left(\beta_{1}, \ldots, \beta_{m} \mid G\right)$ in eq. (2.13) contains the reduced Pfaffian of the $2(m+|G|) \times 2(m+|G|)$ antisymmetric matrix $\Pi$, which can also be written in a block form,

$$
\Pi\left(\tau_{1}, \tau_{2}, \ldots, \tau_{m} \mid G\right)=\left(\begin{array}{cccc}
\mathrm{A}_{G} & \mathrm{~A}_{G, \mathrm{tr}} & -\left(\mathrm{C}_{G}\right)^{T} & -\left(\mathrm{C}_{\mathrm{tr}, G}\right)^{T} \\
\mathrm{~A}_{\mathrm{tr}, G} & \mathrm{~A}_{\mathrm{tr}} & -\left(\mathrm{C}_{G, \mathrm{tr}}\right)^{T} & -\left(\mathrm{C}_{\mathrm{tr}}\right)^{T} \\
\mathrm{C}_{G} & \mathrm{C}_{G, \mathrm{tr}} & \mathrm{B}_{G} & \mathrm{~B}_{G, \mathrm{tr}} \\
\mathrm{C}_{\mathrm{tr}, G} & \mathrm{C}_{\mathrm{tr}} & \mathrm{B}_{\mathrm{tr}, G} & \mathrm{~B}_{\mathrm{tr}}
\end{array}\right),
$$

where the blocks are defined as follows,

- The $|G| \times|G|$ matrix $\mathrm{A}_{G}, \mathrm{~B}_{G}$ and $\mathrm{C}_{G}$ are just those appeared in $\Psi_{G}$ of eq. (A.5).

- The $m \times|G|$ matrix $\mathrm{A}_{\mathrm{tr}, G}, \mathrm{~B}_{\mathrm{tr}, G}$ and $\mathrm{C}_{\mathrm{tr}, G}$ are given by

$$
\left(\mathrm{A}_{\mathrm{tr}, G}\right)_{i b}=\sum_{c \in \tau_{i}} \frac{k_{c} \cdot k_{b}}{\sigma_{c b}}, \quad\left(\mathrm{~B}_{\mathrm{tr}, G}\right)_{i b}=\sum_{c \in \tau_{i}} \frac{\sigma_{c} k_{c} \cdot \epsilon_{b}}{\sigma_{c b}}, \quad\left(\mathrm{C}_{\mathrm{tr}, G}\right)_{i b}=\sum_{c \in \tau_{i}} \frac{\sigma_{c} k_{c} \cdot k_{b}}{\sigma_{c b}} .
$$


- The $|G| \times m$ matrix $\mathrm{A}_{G, \text { tr }}, \mathrm{B}_{G, \text { tr }}$ and $\mathrm{C}_{G, \text { tr }}$ are given by

$$
\mathrm{A}_{G, \mathrm{tr}}=-\left(\mathrm{A}_{\mathrm{tr}, G}\right)^{T}, \quad \mathrm{~B}_{G, \mathrm{tr}}=-\left(\mathrm{B}_{\mathrm{tr}, G}\right)^{T}, \quad\left(\mathrm{C}_{G, \mathrm{tr}}\right)_{a i}=\sum_{c \in \tau_{i}} \frac{\epsilon_{a} \cdot k_{c}}{\sigma_{a c}}
$$

- The $m \times m$ matrix $\mathrm{A}_{\mathrm{tr}}, \mathrm{B}_{\mathrm{tr}}$ and $\mathrm{C}_{\mathrm{tr}}$ are given by

$$
\begin{array}{rlrl}
\left(\mathrm{A}_{\mathrm{tr}}\right)_{i j} & =\sum_{\substack{c \in \tau_{i} \\
d \in \tau_{j}}} \frac{k_{c} \cdot k_{d}}{\sigma_{c d}}, & \left(\mathrm{~B}_{\mathrm{tr}}\right)_{i j}=\sum_{\substack{c \in \tau_{i} \\
d \in \tau_{j}}} \frac{\sigma_{c} \sigma_{d} k_{c} \cdot k_{d}}{\sigma_{c d}}, & \left(\mathrm{C}_{\mathrm{tr}}\right)_{i j}=\sum_{\substack{c \in \tau_{i} \\
d \in \tau_{j}}} \frac{\sigma_{c} k_{c} \cdot k_{d}}{\sigma_{c d}}, \\
\left(\mathrm{~A}_{\mathrm{tr}}\right)_{i i}=0, & \left(\mathrm{~B}_{\mathrm{tr}}\right)_{i i}=0, & \left(\mathrm{C}_{\mathrm{tr}}\right)_{i i}=\frac{1}{2}\left(k_{\tau_{i}}\right)^{2},
\end{array}
$$

where $k_{\tau_{i}}=\sum_{c \in \tau_{i}} k_{c}$ is the total momentum of the trace $\tau_{i}$.

The reduced Pfaffian $\mathrm{Pf}^{\prime}(\Pi)$ is defined as

$$
\begin{array}{rlrl}
\operatorname{Pf}^{\prime}(\Pi) & =(-1)^{i+j} \operatorname{Pf}\left(\Pi_{|G|+i, 2|G|+m+j}^{|G|+i,|G|+m+j}\right) & & 1 \leqslant i, j \leqslant m \\
& =(-1)^{|G|+m-1} \frac{(-1)^{a+b}}{\sigma_{a b}} \operatorname{Pf}\left(\Pi_{a b}^{a b}\right) & a, b \in G \text { and } a<b .
\end{array}
$$

The two expressions are equivalent on the support of the scattering equations when both gluons and traces are present, while the first line applies for the pure scalar case and the second line applies for the pure gluon case. For these two special cases, one can then easily check that eq. (2.15) holds.

To explicitly calculate amplitudes from the CHY formalism, one needs to perform the moduli space integral in eq. (2.1). It turns the delta functions into a Jacobian and results in a summation over the $(n-3)$ ! solutions of the scattering equations. Starting with the gauge fixing (2.3), we get

$$
A_{n}(1,2, \ldots, n)=\mathscr{N} \sum_{\text {solutions }} \frac{\left(\sigma_{1, n-1} \sigma_{n-1, n} \sigma_{n, 1}\right)^{2}}{\operatorname{det}\left(\Phi_{1, n-1, n}^{1, n-1, n}\right)} \operatorname{PT}(1,2, \ldots, n) \mathcal{I}_{n}\left(\beta_{1}, \ldots, \beta_{m} \mid G\right),
$$

where the matrix $\Phi$ is given by

$$
\Phi_{i j}=\frac{k_{i} \cdot k_{j}}{\sigma_{i j}^{2}}, \quad \Phi_{i i}=-\sum_{\substack{j=1 \\ j \neq i}}^{n} \Phi_{i j} .
$$

To obtain the Jacobian $\operatorname{det}\left(\Phi_{1, n-1, n}^{1, n-1, n}\right)$, one needs to delete three rows and columns from $\Phi$ following the gauge fixing. The normalization $\mathscr{N}$ is given by

$$
\mathscr{N}=(-2 i)\left(\frac{g}{\sqrt{2}}\right)^{|G|+2 m-2}\left(-\frac{\lambda}{4}\right)^{|S|-2 m}(-1)^{m},
$$

where $|S|=n-|G|$ is the total number of scalars. This normalization is fixed by comparing with the color-ordered amplitudes from the YMS Lagrangian [57]

$$
\mathcal{L}=-\frac{1}{4}\left(F_{\mu \nu}^{a}\right)^{2}+\frac{1}{2}\left(D_{\mu} \phi^{a A}\right)^{2}+\frac{\lambda}{3 !} f^{a b c} \hat{f}^{A B C} \phi^{a A} \phi^{b B} \phi^{c C}-\frac{g^{2}}{4} f^{a c e} f^{e b d} \phi^{a A} \phi^{b A} \phi^{c B} \phi^{d B}
$$


with the gauge coupling $g$ and $\phi^{3}$ coupling $\lambda$. The scalar $\phi^{a A}$ is in the bi-adjoint representation of the gauge group and another global symmetry group, whose structure constants are $f^{a b c}$ and $\hat{f}^{A B C}$ respectively. In particular, the normalization for the pure gluon cases is

$$
\mathscr{N}=(-2 i)\left(\frac{g}{\sqrt{2}}\right)^{n-2}
$$

which is shared by the two-fermion integrand (2.7) since the two amplitudes are related by a SUSY Ward identity. The outstanding factor 2 can be absorbed into the definition of the reduced Pfaffian, as pointed out in the original CHY paper [25], while the rest simply originate from rescaling color factors. More specifically, we define partial amplitudes in the color trace bases with the normalization $\operatorname{Tr}\left(T^{a} T^{b}\right)=\delta^{a b}$ and $\operatorname{Tr}\left(T^{A} T^{B}\right)=\delta^{A B}$. Both traces are taken in the fundamental representation of the gauge group.

\section{B Mathematica package}

This appendix gives a more in-depth description for the functions provided by our MATHEMATICApackage, IncreasingTrees.m. The package can be found by following this link to our GitLab:

\section{IncreasingTrees GitLab. ${ }^{11}$}

There are no external dependencies for the package, although it does rely on the Graph functionality of MATHEMATICA and thus may depend on the version in use. We have tested the package on MathematiCAversions 11.2 and 12.0.

The package defines a set of objects from which it builds the kinematic numerators, as well as defining construction routines to build the various numerators. It localizes all variables within either its own context IncreasingTrees ' or its private context IncreasingTrees 'Private`, and thus is generally safe to load along other packages. However, it does provide definitions for the single-character variables $k, e, W, d, F$, which may shadow definitions from other packages.

\section{B.1 Constituent objects}

The following objects are used to represent the numerators:

- $\mathrm{k}\left[\mathrm{i} \_\right.$_ $:$Momentum vector for particle(s) (i). Multi-label $\mathrm{k}$ are automatically converted to sums: $\mathrm{k}[1,2]=k_{1}+k_{2}$.

- e[i_ ]: Polarization vector for particle (i)

- $\mathrm{d}\left[\mathrm{x}_{\ldots}, \mathrm{y}_{-}\right]$: Lorentz dot product between two vectors. Orderless, bilinear, and enforces transversality and onshellness of $\mathrm{k}$ and $\mathrm{e}$.

- W[_ ]: Generic baseline factor. Can be swapped out directly using replacement rules for WScalar, WGluon, WFermion2, which are all described in section 2. WL and WR are additionally defined to allow for differentiating theories in the double copy.

\footnotetext{
${ }^{11}$ https://gitlab.com/aedison/increasingtrees.
} 
- WFermion1[i_ $][\quad]$ : Baseline factor for the $\left(-\frac{1}{2},-\frac{1}{2}\right)$ ghost picture fermions in eq. (2.8a). (i) is the gluon in ghost picture -1 . For example, replacing $\mathrm{W} \rightarrow \mathrm{WFermion} 1$ [2] in a numerator will apply this baseline with gluon 2 in the alternate ghost picture.

- spinChain[_]: Abstract spin index contraction generated by WFermions.

- $\chi\left[i_{1}\right]$ : Fermionic wavefunction placeholder for particle (i).

- $\xi\left[i_{\_}\right]$: Ghost picture $-\frac{3}{2}$ fermionic wavefunction for particle (i).

- F[i_ ]: Linearized gluon field-strength. Only appears explicitly in the fermion baseline functions eqs. (2.8a) and (2.8b). We give explicit $\gamma$ contractions in eq. (2.9).

- PT[_ ]: Abstract Parke-Taylor factor used as part of representing (half-)integrands.

- cf [ ] ]: Abstract color factor for color-dressed amplitude construction, following eq. (1.2).

\section{B.2 Construction functions}

The package exposes the following functions used in assembling half-ladder numerators:

- increasingTrees[labels_List]: Constructs all ordered trees where the vertex labels are taken from (labels) and the ordering is consistent with the ordering of (labels). Thus, each tree spans the vertices and is only composed of DirectedEdges of the form (labels $)[[\mathrm{j}]] \rightarrow($ labels $)[[\mathrm{i}]]$ with $i<j$.

- itNumer[g Graph, maxV_ ]: Processes (g) to extract the baseline information and runs the recursive construction (detailed in section 3.2) starting from each vertex along the baseline to generate a kinematic polynomial. (maxv) specifies which vertex should be treated as the end of the baseline.

- ptTreeNumer[points_Integer, onlyFuncQ_:False]: This function has a different return scheme based on whether (onlyFuncQ) is True or False:

- True: generates a Function with (points) slots that in turn produces a halfladder numerator with the specified external labels.

- False(default): generates all half-ladder numerators with (points) number of external particles, dressed with appropriate PT factors. Explicitly uses the crossing symmetry of labels $(2, \ldots$, (points) -1$)$.

- multiTraceNumer[labels_List, traces_List]: Computes the kinematic numerator for a mix of scalars and gluons carrying labels specified and ordered in (labels), grouped into scalar traces specified by (traces). Handles the construction and insertion of the baseline factor directly. Traces should be given as a List of Lists, where each sublist specifies a color-ordered trace of scalar particles. Gluons can either be specified as length 1 sublists in (traces), or omitted from the trace specification entirely. Expects each particle to be labeled the same way in (labels) and (traces). 
- multiTraceIntegrand[points_Integer, traces_List]: Computes the explicit permutation sum of multiTraceNumer over the permutations of labels ranging from $(2, \ldots$, (points) -1$)$, using the trace configurations given in (traces), and dressed with appropriate PT factors. The particle labels in (traces) should be given as integers $1, \ldots$, (points).

Unfortunately, since the symmetries of the multi-trace numerators are significantly more nuanced than in the case of only gluons (or gluons with two scalars or two fermions), we don't make direct use of them in multiTraceIntegrand like we do in ptTreeNumer. As such, while multiTraceIntegrand and ptTreeNumer produce identical results for pure gluons, we strongly recommend to use ptTreeNumer in these situations, especially for larger particle number.

In addition to the half-ladder numerator constructors, we also provide the tools to assemble the numerators into amplitudes. However, these computations run into the $\mathcal{O}((n-$ $\left.2) !^{2}\right)$ complexity of calculating the full matrtix of $m(\alpha \mid \beta)$.

- $\operatorname{mab}[\alpha$ List, $\beta$ List $]:$ Computes the biadjoint scalar amplitude for orderings $(\alpha)$ and $(\beta)$. The orderings should be in the same KK basis: $\alpha_{1}=\beta_{1}$ and $\alpha_{n}=\beta_{n}$. The momentum $k_{\alpha_{n}}$ is implicitly removed via momentum conservation. Uses the algorithm proposed by ref. [89].

- Aamp[labels_List]: Constructs the Yang-Mills tree amplitude according to eq. (1.2), where the external particle labels are drawn from (labels). Includes the explicit normalization factor eq. (A.13). The baseline factors $W[\ldots]$ are left unevaluated to allow for choice of special particles.

- Aamp[n_Integer ]: Constructs the YM tree amplitude using $1, \ldots, n$ as the labels.

- Mamp[labels_List]: Constructs the gravity tree amplitude according to eq. (1.3), where the external particle labels are drawn from (labels). The baseline factors for the two copies are left unevaluated, with seperate labels WL and WR to allow for double copies between different types of special particles. No explicit normalization is used.

- Mamp[n_Integer ]: Constructs the gravity tree amplitude using $1, \ldots, n$ as the labels.

It is important to note that only Aamp includes explicit normalizations. However, we make the YMS normalization accessible via

- ymsNorm[traces_List ]: Computes the YMS normalization $\mathscr{N}$ from eq. (A.13), with the trace groupings specified as seperate Lists in (traces). Gluons/fermions are specified via length-one Lists. ymCoup and scCoup are used to represent the sYM coupling $g$ and scalar coupling $\lambda$, respectively.

Open Access. This article is distributed under the terms of the Creative Commons Attribution License (CC-BY 4.0), which permits any use, distribution and reproduction in any medium, provided the original author(s) and source are credited. 


\section{References}

[1] Z. Bern, J.J.M. Carrasco and H. Johansson, New Relations for Gauge-Theory Amplitudes, Phys. Rev. D 78 (2008) 085011 [arXiv:0805.3993] [InSPIRE].

[2] Z. Bern, J.J.M. Carrasco and H. Johansson, Perturbative Quantum Gravity as a Double Copy of Gauge Theory, Phys. Rev. Lett. 105 (2010) 061602 [arXiv:1004.0476] [INSPIRE].

[3] Z. Bern, J.J.M. Carrasco, M. Chiodaroli, H. Johansson and R. Roiban, The Duality Between Color and Kinematics and its Applications, arXiv:1909.01358 [INSPIRE].

[4] H. Johansson, G. Kälin and G. Mogull, Two-loop supersymmetric QCD and half-maximal supergravity amplitudes, JHEP 09 (2017) 019 [arXiv: 1706.09381] [INSPIRE].

[5] Z. Bern et al., Ultraviolet Properties of $\mathcal{N}=8$ Supergravity at Five Loops, Phys. Rev. D 98 (2018) 086021 [arXiv: 1804.09311] [INSPIRE].

[6] Z. Bern, S. Davies and T. Dennen, Enhanced ultraviolet cancellations in $\mathcal{N}=5$ supergravity at four loops, Phys. Rev. D 90 (2014) 105011 [arXiv:1409.3089] [INSPIRE].

[7] Z. Bern, S. Davies, T. Dennen, A.V. Smirnov and V.A. Smirnov, Ultraviolet Properties of $N=4$ Supergravity at Four Loops, Phys. Rev. Lett. 111 (2013) 231302 [arXiv:1309.2498] [INSPIRE].

[8] A. Luna, R. Monteiro, I. Nicholson, D. O'Connell and C.D. White, The double copy: Bremsstrahlung and accelerating black holes, JHEP 06 (2016) 023 [arXiv:1603.05737] [INSPIRE].

[9] W.D. Goldberger and A.K. Ridgway, Radiation and the classical double copy for color charges, Phys. Rev. D 95 (2017) 125010 [arXiv:1611.03493] [INSPIRE].

[10] A. Luna, I. Nicholson, D. O'Connell and C.D. White, Inelastic Black Hole Scattering from Charged Scalar Amplitudes, JHEP 03 (2018) 044 [arXiv: 1711.03901] [INSPIRE].

[11] C.-H. Shen, Gravitational Radiation from Color-Kinematics Duality, JHEP 11 (2018) 162 [arXiv: 1806.07388] [INSPIRE].

[12] J. Plefka, J. Steinhoff and W. Wormsbecher, Effective action of dilaton gravity as the classical double copy of Yang-Mills theory, Phys. Rev. D 99 (2019) 024021 [arXiv: 1807.09859] [INSPIRE].

[13] C. Cheung, I.Z. Rothstein and M.P. Solon, From Scattering Amplitudes to Classical Potentials in the Post-Minkowskian Expansion, Phys. Rev. Lett. 121 (2018) 251101 [arXiv: 1808.02489] [INSPIRE].

[14] D.A. Kosower, B. Maybee and D. O'Connell, Amplitudes, Observables, and Classical Scattering, JHEP 02 (2019) 137 [arXiv: 1811.10950] [INSPIRE].

[15] A. Guevara, A. Ochirov and J. Vines, Scattering of Spinning Black Holes from Exponentiated Soft Factors, JHEP 09 (2019) 056 [arXiv: 1812.06895] [INSPIRE].

[16] Z. Bern, C. Cheung, R. Roiban, C.-H. Shen, M.P. Solon and M. Zeng, Scattering Amplitudes and the Conservative Hamiltonian for Binary Systems at Third Post-Minkowskian Order, Phys. Rev. Lett. 122 (2019) 201603 [arXiv:1901.04424] [InSPIRE].

[17] J. Plefka, C. Shi, J. Steinhoff and T. Wang, Breakdown of the classical double copy for the effective action of dilaton-gravity at NNLO, Phys. Rev. D 100 (2019) 086006 [arXiv: 1906.05875] [INSPIRE].

[18] B. Maybee, D. O'Connell and J. Vines, Observables and amplitudes for spinning particles and black holes, JHEP 12 (2019) 156 [arXiv: 1906.09260] [INSPIRE]. 
[19] A. Guevara, A. Ochirov and J. Vines, Black-hole scattering with general spin directions from minimal-coupling amplitudes, Phys. Rev. D 100 (2019) 104024 [arXiv:1906.10071] [INSPIRE].

[20] H. Johansson and A. Ochirov, Double copy for massive quantum particles with spin, JHEP 09 (2019) 040 [arXiv: 1906.12292] [INSPIRE].

[21] Z. Bern, C. Cheung, R. Roiban, C.-H. Shen, M.P. Solon and M. Zeng, Black Hole Binary Dynamics from the Double Copy and Effective Theory, JHEP 10 (2019) 206 [arXiv: 1908.01493] [INSPIRE].

[22] Y.F. Bautista and A. Guevara, On the Double Copy for Spinning Matter, arXiv:1908.11349 [INSPIRE].

[23] J. Plefka, C. Shi and T. Wang, Double copy of massive scalar QCD, Phys. Rev. D 101 (2020) 066004 [arXiv: 1911.06785] [inSPIRE].

[24] F. Cachazo, S. He and E.Y. Yuan, Scattering equations and Kawai-Lewellen-Tye orthogonality, Phys. Rev. D 90 (2014) 065001 [arXiv:1306.6575] [InSPIRE].

[25] F. Cachazo, S. He and E.Y. Yuan, Scattering of Massless Particles in Arbitrary Dimensions, Phys. Rev. Lett. 113 (2014) 171601 [arXiv:1307.2199] [InSPIRE].

[26] F. Cachazo, S. He and E.Y. Yuan, Scattering of Massless Particles: Scalars, Gluons and Gravitons, JHEP 07 (2014) 033 [arXiv: 1309.0885] [INSPIRE].

[27] F. Cachazo, S. He and E.Y. Yuan, Einstein-Yang-Mills Scattering Amplitudes From Scattering Equations, JHEP 01 (2015) 121 [arXiv:1409.8256] [INSPIRE].

[28] F. Cachazo, S. He and E.Y. Yuan, Scattering Equations and Matrices: From Einstein To Yang-Mills, DBI and NLSM, JHEP 07 (2015) 149 [arXiv:1412.3479] [INSPIRE].

[29] S. He and Y. Zhang, New Formulas for Amplitudes from Higher-Dimensional Operators, JHEP 02 (2017) 019 [arXiv: 1608.08448] [INSPIRE].

[30] T. Azevedo and O.T. Engelund, Ambitwistor formulations of $R^{2}$ gravity and $(D F)^{2}$ gauge theories, JHEP 11 (2017) 052 [arXiv:1707.02192] [INSPIRE].

[31] F. Cachazo, A. Guevara, M. Heydeman, S. Mizera, J.H. Schwarz and C. Wen, The S Matrix of $6 D$ Super Yang-Mills and Maximal Supergravity from Rational Maps, JHEP 09 (2018) 125 [arXiv: 1805.11111] [INSPIRE].

[32] S. He, F. Teng and Y. Zhang, String amplitudes from field-theory amplitudes and vice versa, Phys. Rev. Lett. 122 (2019) 211603 [arXiv: 1812.03369] [InSPIRE].

[33] Y. Geyer and L. Mason, Polarized Scattering Equations for 6D Superamplitudes, Phys. Rev. Lett. 122 (2019) 101601 [arXiv: 1812.05548] [INSPIRE].

[34] S. He, F. Teng and Y. Zhang, String Correlators: Recursive Expansion, Integration-by-Parts and Scattering Equations, JHEP 09 (2019) 085 [arXiv: 1907.06041] [INSPIRE].

[35] L. Mason and D. Skinner, Ambitwistor strings and the scattering equations, JHEP 07 (2014) 048 [arXiv: 1311.2564$]$ [INSPIRE].

[36] N. Berkovits, Infinite Tension Limit of the Pure Spinor Superstring, JHEP 03 (2014) 017 [arXiv:1311.4156] [INSPIRE].

[37] T. Adamo, E. Casali and D. Skinner, Ambitwistor strings and the scattering equations at one loop, JHEP 04 (2014) 104 [arXiv: 1312.3828] [INSPIRE].

[38] T. Adamo and E. Casali, Scattering equations, supergravity integrands, and pure spinors, JHEP 05 (2015) 120 [arXiv:1502.06826] [INSPIRE]. 
[39] E. Casali, Y. Geyer, L. Mason, R. Monteiro and K.A. Roehrig, New Ambitwistor String Theories, JHEP 11 (2015) 038 [arXiv: 1506.08771] [INSPIRE].

[40] T. Azevedo and R.L. Jusinskas, Connecting the ambitwistor and the sectorized heterotic strings, JHEP 10 (2017) 216 [arXiv:1707.08840] [INSPIRE].

[41] T. Azevedo, R.L. Jusinskas and M. Lize, Bosonic sectorized strings and the $(D F)^{2}$ theory, JHEP 01 (2020) 082 [arXiv:1908.11371] [INSPIRE].

[42] V. Del Duca, L.J. Dixon and F. Maltoni, New color decompositions for gauge amplitudes at tree and loop level, Nucl. Phys. B 571 (2000) 51 [hep-ph/9910563] [INSPIRE].

[43] C.R. Mafra, O. Schlotterer and S. Stieberger, Explicit BCJ Numerators from Pure Spinors, JHEP 07 (2011) 092 [arXiv: 1104.5224] [inSPIRE].

[44] J. Broedel, O. Schlotterer and S. Stieberger, Polylogarithms, Multiple Zeta Values and Superstring Amplitudes, Fortsch. Phys. 61 (2013) 812 [arXiv:1304.7267] [INSPIRE].

[45] Y.-t. Huang, O. Schlotterer and C. Wen, Universality in string interactions, JHEP 09 (2016) 155 [arXiv: 1602.01674] [INSPIRE].

[46] S. Mizera, Combinatorics and Topology of Kawai-Lewellen-Tye Relations, JHEP 08 (2017) 097 [arXiv: 1706 . 08527] [INSPIRE].

[47] S. Mizera, Scattering Amplitudes from Intersection Theory, Phys. Rev. Lett. 120 (2018) 141602 [arXiv: 1711.00469] [InSPIRE].

[48] T. Azevedo, M. Chiodaroli, H. Johansson and O. Schlotterer, Heterotic and bosonic string amplitudes via field theory, JHEP 10 (2018) 012 [arXiv:1803. 05452] [INSPIRE].

[49] Z. Bern, T. Dennen, Y.-t. Huang and M. Kiermaier, Gravity as the Square of Gauge Theory, Phys. Rev. D 82 (2010) 065003 [arXiv:1004.0693] [InSPIRE].

[50] D. Vaman and Y.-P. Yao, Constraints and Generalized Gauge Transformations on Tree-Level Gluon and Graviton Amplitudes, JHEP 11 (2010) 028 [arXiv:1007.3475] [INSPIRE].

[51] Y.-J. Du, B. Feng and C.-H. Fu, BCJ Relation of Color Scalar Theory and KLT Relation of Gauge Theory, JHEP 08 (2011) 129 [arXiv:1105.3503] [INSPIRE].

[52] N.E.J. Bjerrum-Bohr, P.H. Damgaard, R. Monteiro and D. O'Connell, Algebras for Amplitudes, JHEP 06 (2012) 061 [arXiv: 1203.0944] [INSPIRE].

[53] H. Kawai, D.C. Lewellen and S.H.H. Tye, A Relation Between Tree Amplitudes of Closed and Open Strings, Nucl. Phys. B 269 (1986) 1 [INSPIRE].

[54] M. Kiermaier, Gravity as the Square of Gauge Theory, talk given at Amplitudes 2010, QMUL, London, U.K, 4-7 May 2010 and online pdf version at https://strings.ph.qmul.ac.uk/ theory/Amplitudes2010/Talks/MK2010.pdf.

[55] N.E.J. Bjerrum-Bohr, P.H. Damgaard, T. Søndergaard and P. Vanhove, The Momentum Kernel of Gauge and Gravity Theories, JHEP 01 (2011) 001 [arXiv:1010.3933] [InSPIRE].

[56] N.E.J. Bjerrum-Bohr, J.L. Bourjaily, P.H. Damgaard and B. Feng, Manifesting Color-Kinematics Duality in the Scattering Equation Formalism, JHEP 09 (2016) 094 [arXiv: 1608.00006] [INSPIRE].

[57] M. Chiodaroli, M. Günaydin, H. Johansson and R. Roiban, Scattering amplitudes in $\mathcal{N}=2$ Maxwell-Einstein and Yang-Mills/Einstein supergravity, JHEP 01 (2015) 081 [arXiv: 1408.0764] [INSPIRE].

[58] S. Stieberger and T.R. Taylor, New relations for Einstein-Yang-Mills amplitudes, Nucl. Phys. B 913 (2016) 151 [arXiv: 1606.09616] [INSPIRE]. 
[59] D. Nandan, J. Plefka, O. Schlotterer and C. Wen, Einstein-Yang-Mills from pure Yang-Mills amplitudes, JHEP 10 (2016) 070 [arXiv:1607.05701] [INSPIRE].

[60] L. de la Cruz, A. Kniss and S. Weinzierl, Relations for Einstein-Yang-Mills amplitudes from the CHY representation, Phys. Lett. B 767 (2017) 86 [arXiv:1607.06036] [InSPIRE].

[61] O. Schlotterer, Amplitude relations in heterotic string theory and Einstein-Yang-Mills, JHEP 11 (2016) 074 [arXiv:1608.00130] [INSPIRE].

[62] C.-H. Fu, Y.-J. Du, R. Huang and B. Feng, Expansion of Einstein-Yang-Mills Amplitude, JHEP 09 (2017) 021 [arXiv: 1702.08158] [INSPIRE].

[63] M. Chiodaroli, M. Günaydin, H. Johansson and R. Roiban, Explicit Formulae for Yang-Mills-Einstein Amplitudes from the Double Copy, JHEP 07 (2017) 002 [arXiv: 1703.00421] [INSPIRE].

[64] F. Teng and B. Feng, Expanding Einstein-Yang-Mills by Yang-Mills in CHY frame, JHEP 05 (2017) 075 [arXiv: 1703.01269] [INSPIRE].

[65] Y.-J. Du and F. Teng, BCJ numerators from reduced Pfaffian, JHEP 04 (2017) 033 [arXiv: 1703.05717] [INSPIRE].

[66] Y.-J. Du, B. Feng and F. Teng, Expansion of All Multitrace Tree Level EYM Amplitudes, JHEP 12 (2017) 038 [arXiv:1708.04514] [INSPIRE].

[67] B. Feng, X. Li and K. Zhou, Expansion of Einstein-Yang-Mills theory by differential operators, Phys. Rev. D 100 (2019) 125012 [arXiv:1904.05997] [INSPIRE].

[68] Y. Geyer, L. Mason, R. Monteiro and P. Tourkine, Loop Integrands for Scattering Amplitudes from the Riemann Sphere, Phys. Rev. Lett. 115 (2015) 121603 [arXiv:1507.00321] [INSPIRE].

[69] Y. Geyer, L. Mason, R. Monteiro and P. Tourkine, One-loop amplitudes on the Riemann sphere, JHEP 03 (2016) 114 [arXiv:1511.06315] [INSPIRE].

[70] Y. Geyer, L. Mason, R. Monteiro and P. Tourkine, Two-Loop Scattering Amplitudes from the Riemann Sphere, Phys. Rev. D 94 (2016) 125029 [arXiv:1607.08887] [InSPIRE].

[71] F. Cachazo, S. He and E.Y. Yuan, One-Loop Corrections from Higher Dimensional Tree Amplitudes, JHEP 08 (2016) 008 [arXiv:1512.05001] [INSPIRE].

[72] S. He and E.Y. Yuan, One-loop Scattering Equations and Amplitudes from Forward Limit, Phys. Rev. D 92 (2015) 105004 [arXiv:1508.06027] [INSPIRE].

[73] S. He and O. Schlotterer, New Relations for Gauge-Theory and Gravity Amplitudes at Loop Level, Phys. Rev. Lett. 118 (2017) 161601 [arXiv:1612.00417] [INSPIRE].

[74] S. He, O. Schlotterer and Y. Zhang, New BCJ representations for one-loop amplitudes in gauge theories and gravity, Nucl. Phys. B 930 (2018) 328 [arXiv:1706.00640] [InSPIRE].

[75] Y. Geyer and R. Monteiro, Gluons and gravitons at one loop from ambitwistor strings, JHEP 03 (2018) 068 [arXiv:1711.09923] [INSPIRE].

[76] J. Agerskov, N.E.J. Bjerrum-Bohr, H. Gomez and C. Lopez-Arcos, One-Loop Yang-Mills Integrands from Scattering Equations, Phys. Rev. D 102 (2020) 045023 [arXiv:1910.03602] [INSPIRE].

[77] C.R. Mafra and O. Schlotterer, Berends-Giele recursions and the BCJ duality in superspace and components, JHEP 03 (2016) 097 [arXiv:1510.08846] [INSPIRE].

[78] A. Edison, S. He, O. Schlotterer and F. Teng, One-loop Correlators and BCJ Numerators from Forward Limits, JHEP 09 (2020) 079 [arXiv:2005.03639] [INSPIRE]. 
[79] C.S. Lam and Y.-P. Yao, Evaluation of the Cachazo-He-Yuan gauge amplitude, Phys. Rev. D 93 (2016) 105008 [arXiv: 1602.06419] [INSPIRE].

[80] C. Cheung and D. O'Connell, Amplitudes and Spinor-Helicity in Six Dimensions, JHEP 07 (2009) 075 [arXiv: 0902.0981] [INSPIRE].

[81] R. Kleiss and H. Kuijf, Multi-Gluon Cross-sections and Five Jet Production at Hadron Colliders, Nucl. Phys. B 312 (1989) 616 [inSPIRE].

[82] X. Gao, S. He and Y. Zhang, Labelled tree graphs, Feynman diagrams and disk integrals, JHEP 11 (2017) 144 [arXiv:1708.08701] [INSPIRE].

[83] M. Chiodaroli, M. Günaydin, H. Johansson and R. Roiban, Spontaneously Broken Yang-Mills-Einstein Supergravities as Double Copies, JHEP 06 (2017) 064 [arXiv: 1511.01740] [INSPIRE].

[84] Z. Bern, J.J.M. Carrasco, T. Dennen, Y.-t. Huang and H. Ita, Generalized Unitarity and Six-Dimensional Helicity, Phys. Rev. D 83 (2011) 085022 [arXiv:1010.0494] [InSPIRE].

[85] N. Arkani-Hamed, T.-C. Huang and Y.-t. Huang, Scattering Amplitudes For All Masses and Spins, arXiv: 1709.04891 [INSPIRE].

[86] A. Ochirov, Helicity amplitudes for QCD with massive quarks, JHEP 04 (2018) 089 [arXiv: 1802.06730] [INSPIRE].

[87] D. Gerosa and M. Vallisneri, filltex: Automatic queries to ADS and INSPIRE databases to fill LaTeX bibliography, J. Open Source Software 2 (2017) 222.

[88] https://en.wikipedia.org/wiki/Pfaffian.

[89] C.R. Mafra, Berends-Giele recursion for double-color-ordered amplitudes, JHEP 07 (2016) 080 [arXiv: 1603.09731] [INSPIRE]. 